\title{
Monopolistic Supply of Sorting, Inequality and Welfare
}

\section{Max Planck Institute for Tax Law and Public Finance}

Working Paper 2018 - 15

November 2018

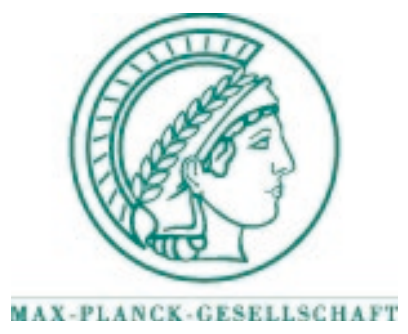

Max Planck Institute for

Tax Law and Public Finance

Department of Business and Tax Law

Department of Public Economics

http:/ / www.tax.mpg.de 
Working papers of the Max Planck Institute for Tax Law and Public Finance Research Paper Series serve to disseminate the research results of work in progress prior to publication to encourage the exchange of ideas and academic debate. Inclusion of a paper in the Research Paper Series does not constitute publication and should not limit publication in any other venue. The preprints published by the Max Planck Institute for Tax Law and Public Finance represent the views of the respective author(s) and not of the Institute as a whole. Copyright remains with the author(s).

Max Planck Institute for Tax Law and Public Finance

Marstallplatz 1

D-80539 Munich

Tel: $\quad+498924246-0$

Fax: $\quad+498924246-501$

E-mail: ssrn@tax.mpg.de

http://www.tax.mpg.de 


\title{
Monopolistic Supply of Sorting, Inequality and Welfare*
}

\author{
Lisa Windsteiger ${ }^{\dagger}$
}

\begin{abstract}
In this paper I present a model in which a monopolist offers citizens the opportunity to segregate into groups according to income. I focus initially on the case of two groups and show that a monopolist with fixed costs of offering the sorting technology will see profits increase as income inequality increases. I then analyze how the monopolist's optimal group partition varies with inequality and show that for a broad field of income distributions, monopolist profits increase with inequality, while at the same time total welfare of sorting given the monopolist's optimal schedule decreases. In the last section I examine how these findings generalize if the monopolist doesn't face costs of offering the sorting technology and can therefore offer as many groups as she wants.
\end{abstract}

JEL Classification: D83, D85, Z13

Keywords: Stratification, Assortative Matching, Group Formation

*I thank Ronny Razin, Matt Levy and the participants of the LSE Microeconomic Theory Work in Progress Seminar for their helpful suggestions and comments.

${ }^{\dagger}$ Max Planck Institute for Tax Law and Public Finance, Email: Lisa.Windsteiger@tax.mpg.de 


\section{Introduction}

In recent years, we have observed a rise in social segregation in many industrialized countries. People tend to interact increasingly with others who are not too different from themselves in terms of income, education and political beliefs. ${ }^{1}$ Moreover, evidence suggests that segregation and income inequality tend to move jointly. Several studies for the US show that both income inequality and segregation have increased in most metropolitan areas over the past 40 years. $^{2}$

The reasons for this co-movement haven't been explored widely so far. While the presence of assortative matching and (positive) sorting has been extensively discussed in the economics and sociology literature, little research has been done so far on the supply side of segregation and the relationship between inequality and the supply of segregation.

Given the trend of mounting social segregation, an important question is also the social desirability of sorting. If people benefit from interacting with wealthy and influential people, poor people who are deprived of these contacts due to social seclusion will suffer. But sorting might not be universally beneficial for the rich either: Especially if inequality is high, it might be the case that they have to pay huge sums to separate themselves off from the rest of society (e.g. via gated communities or private schools). While Becker (1974) shows that assortative matching always maximizes total surplus in society, Levy and Razin (2015) and Hoppe et al. (2009) demonstrate that segregation is not necessarily beneficial for welfare if we count these "sorting fees" as deadweight loss and subtract them from the surplus.

Finally, it is important to note that the interests of a supplier of the sorting technology might be different from society's interests, and that the way sorting is implemented need not be optimal for society. In addition, an increase in inequality is likely to have different effects on the supplier of the sorting technology and on welfare.

In the present paper, I make a first attempt to analyze the relationship between income inequality and the supply of sorting and to examine how

\footnotetext{
${ }^{1}$ See e.g. Forman and Koch (2013) and Bishop (2008) for evidence on the US.

${ }^{2}$ See e.g. Reardon and Bischoff (2011) and Watson (2009).
} 
well the interests of the supplier of the sorting technology and of society as a whole are aligned, especially in the face of rising inequality.

In my analysis, I deploy a simple model in which income is distributed unequally in society and people can pay a "fee" to join a group and interact only with members of that group henceforth. I examine how this fee will be set if a profit-maximizing monopolist offers this sorting technology, and I analyze the monopolist's profits and society's total welfare resulting from this split into groups. I show that an increase in inequality increases monopolist profits from offering people the possibility to segregate, and potentially also welfare from segregation. However, I demonstrate that there is often a conflict between welfare and monopolist profits, in the sense that different partitions of society would be optimal for profits and welfare the way in which the monopolist splits up society is in general not efficient (i.e. welfare maximizing). This conflict tends to intensify as inequality increases: monopolist profits increase, while welfare from sorting decreases as income inequality climbs high. At the end of the paper I argue that there is a sense in which this finding holds also if we allow the monopolist to offer more than just one group.

The rest of the paper is organized as follows: Section 2 presents related literature, Section 3 introduces the model of sorting according to income and examines how changes in inequality affect monopolist profits and welfare. Section 4 uses a stylized income distribution (the symmetric atom distribution) to demonstrate that there can be a conflict between monopolist profits and welfare as inequality increases, and generalizes this result to other types of income distributions. Section 5 examines the effect of increasing inequality on monopolist profits and welfare if the monopolist can offer as many cutoffs as she wants and Section 6 concludes.

\section{Related Literature}

The standard model of sorting and assortative matching is outlined and analyzed in Becker (1974). Levy and Razin (2015) examine total welfare and preferences for redistribution in the presence of costly income sorting without explicitly modelling the supply side of the sorting technology. Rayo (2013) characterizes optimal sorting if a profit-maximizing monop- 
olist without costs chooses the sorting schedule, while Damiano and Li (2007) analyze the case of two or more competing firms. My paper carries elements of both Levy and Razin (2015) (in the sense that I analyze the normative aspects of segregation, in particular its effects on welfare) and of Rayo (2013) (because I assume that the sorting technology is offered by a profit-maximizing monopolist). The main contribution of my paper is that I examine how optimal sorting varies with inequality and how this affects the (potential) conflict between welfare and monopolist profit.

My paper is also related to the literature of costly signalling (see e.g. Hoppe, Moldovanu and Sela (2009)) and conspicuous consumption (see e.g. Pesendorfer (1995), Bagwell and Bernheim (1996) and Veblen (1899)) and to the literature of educational segregation via private schools (see e.g. Fernandez and Rogerson (2003), Epple and Romano (1998) and Levy and Razin (2016)).

\section{Inequality, monopolist profit and welfare}

Let income $y$ in an economy be distributed according to an income distribution $F(y)$, on the interval $Y=\left[0, y_{\max }\right]$ (where $y_{\max }<\infty$ unless explicitly mentioned otherwise). Assume furthermore that $F(y)$ is continuous and strictly monotonic. Suppose that an agent's utility is increasing not only in her own income but also in the average income of the people that she interacts with, which I will henceforth call her "reference group". Specifically, a person with income $y_{j}$ gets utility $U_{j}=y_{j} E\left(y \mid y \in S_{i}\right)$, where $S_{i}$ is individual $j$ 's reference group. If there is no economic segregation, everybody's reference group is a representative sample of the whole population, such that $U_{j}=y_{j} E(y)$. However, a person with income $y_{j}$ can pay a fee $b>0$ to join group $S_{b}$ and get utility

$$
y_{j} E\left[y \mid y \in S_{b}\right]-b
$$

or refrain from paying $b$ and get

$$
y_{j} E\left[y \mid y \in S_{0}\right]
$$


where $S_{b}$ is the set of incomes $y$ of people who have paid $b$ and $S_{0}$ is the set of incomes $y$ of people who haven't paid $b$. Then I can define the following:

Definition 1 A sorting equilibrium is a partition $\left[S_{0}, S_{b}\right]$ of $Y$ and a sorting fee $b>0$ such that

$$
\begin{aligned}
& y E\left[y \mid y \in S_{b}\right]-b \leq y E\left[y \mid y \in S_{0}\right] \quad \forall y \in S_{0} \\
& y E\left[y \mid y \in S_{b}\right]-b \geq y E\left[y \mid y \in S_{0}\right] \quad \forall y \in S_{b}
\end{aligned}
$$

In a sorting equilibrium as defined above people stay in the group that gives them the highest utility.

In Windsteiger (2018), I discuss this model in detail and show that in any sorting equilibrium, group $S_{b}$ must have a higher average income than group $S_{0}$, and that all sorting equilibria will be monotone, meaning that the groups $S_{0}$ and $S_{b}$ are single intervals of $Y$ (where group $S_{b}$ must lie to the right of group $S_{0}$ on the $Y$ scale).

Therefore, I will from now on call people in $S_{b}$ "the rich" and people in $S_{0}$ "the poor". Furthermore, the fact that all equilibria are monotone allows me to rewrite the definition of a sorting equilibrium in terms of a cutoff $\hat{y}$, where everybody with income below the cutoff is in the poor group and everybody with income above the cutoff is in the rich group. For simplicity of notation I will denote average income in the rich group, $E\left[y \mid y \in S_{b}\right]$, by $\bar{E}(\hat{y})$ and average income in the poor group, $E\left[y \mid y \in S_{0}\right]$, by $\underline{E}(\hat{y})$. In Windsteiger (2018) I show the following:

Corollary 1 A sorting equilibrium is characterized by a cutoff $\hat{y} \in Y$ and a sorting fee $b$ such that

$$
\hat{y} \bar{E}(\hat{y})-\hat{y} \underline{E}(\hat{y})=b
$$

A person with income $\hat{y}$ just at the border of the two groups $S_{b}$ and $S_{0}$ has to be exactly indifferent between joining either of the two groups in equilibrium. For the remainder of the paper I will choose the convention that people with income $\hat{y}$ (who are indifferent between the two groups) stay in the poor group.

It can immediately be seen from (3) that the sorting fee is uniquely determined by the equilibrium cutoff $\hat{y}$, i.e. for a given equilibrium partition 
$\left\{[0, \hat{y}],\left(\hat{y}, y_{\max }\right]\right\}$, the sorting fee $b$ is unique. The reverse statement is not true in general: For a given $b$, there might be multiple cutoffs $\hat{y}$ that satisfy $\hat{y}(\bar{E}(\hat{y})-\underline{E}(\hat{y}))=b$ (this could happen if the distribution is such that $\hat{y}(\bar{E}(\hat{y})-\underline{E}(\hat{y}))$ is not strictly increasing or decreasing for all $\left.\hat{y} \in Y^{3}\right)$. For a given sorting fee, there could therefore be several monotone partitions of society that would be sorting equilibria given this fee. When I model the supply side below, I thus require that whoever offers the sorting technology chooses the cutoff optimally and I implicitly assume that the supplier can then ensure that the agents coordinate on the equilibrium that yields the highest payoff for the supplier (which, in the case of a profit-maximizing firm, would always be the lowest cutoff $\hat{y}$ such that $\hat{y}(\bar{E}(\hat{y})-\underline{E}(\hat{y}))=b$, because it yields the largest mass of customers).

\subsection{Monopolist profit}

The model outlined above shows how the sorting fee has to be set in order to generate a certain partition of society. But who determines how the groups in society look like? Who offers the sorting technology and chooses the cutoff?

For the remainder of this paper I will assume that the sorting technology is offered by a profit-maximizing monopolist and I will examine the implications of an increase in inequality for the monopolist's profits and for total welfare. In the next sections I will focus on the model of sorting with two groups as described above. The monopolist can therefore only decide between offering one cutoff or staying inactive, but she cannot offer more than one cutoff. This could be modelled explicitly by assuming that the costs of offering more than one cutoff are prohibitively high. In the last section of this paper, I will discuss what happens if the monopolist's costs are negligible and she can therefore offer as many cutoffs as she wants.

If the monopolist faces fixed costs $c>0$ of operating, her profits from offering sorting are

$$
\Pi\left(\hat{y}^{*}\right)=R\left(\hat{y}^{*}\right)-c,
$$

\footnotetext{
${ }^{3}$ It can be shown that a sufficient condition for $\hat{y}(\bar{E}-\underline{E})$ to be monotone is that the income distribution is new worse than used in expectations (NWUE). For a definition of the NWUE property see Section 3.2.
} 
where $R\left(\hat{y}^{*}\right)$ is the revenue from offering sorting at cutoff $\hat{y}^{*}$ and $\hat{y}^{*}$ is placed optimally,

$$
\hat{y}^{*}=\underset{\hat{y}}{\arg \max } R(\hat{y}) .
$$

Revenue at cutoff $\hat{y}$ is given by

$$
R(\hat{y})=\hat{y}(\bar{E}(\hat{y})-\underline{E}(\hat{y}))(1-F(\hat{y}))=\hat{y}(E-\underline{E}(\hat{y})) .
$$

It is straightforward to see that the solution to the revenue maximization problem must be interior, because $R(0)=R\left(y_{\max }\right)=0$ whereas $R(\hat{y})$ is strictly positive for any interior $\hat{y}$.

Suppose that the income distribution and the fixed costs $c$ are such that $\Pi\left(\hat{y}^{*}\right)>0$ and hence it is profitable for the monopolist to offer the sorting technology. What happens to her profits as inequality increases? In the following, I will show that the monopolist's profits always rise if inequality increases in the form of a particular type of mean-preserving spread of the income distribution. I shall say that a mean-preserving spread is monotone if $\bar{E}(\hat{y})$ increases and $\underline{E}(\hat{y})$ decreases for any interior cutoff $\hat{y}$ (while of course, as implied by the definition of a mean-preserving spread, average income $E$ doesn't change.)

Proposition 1 A monotone mean-preserving spread of the income distribution increases the monopolist's profits from offering sorting.

Proof. If inequality increases in the form of a monotone mean-preserving spread of the income distribution, the difference $E-\underline{E}$ will increase. This implies a rise in $\hat{y}^{*}(E-\underline{E})$, keeping $\hat{y}^{*}$ constant at the optimal choice for the initial income distribution. It is very likely that the optimal cutoff will also change for the monopolist, but even with keeping the old cutoff, her revenues increase, and they will do even more so if the monopolist also chooses the cutoff optimally.

Remark 1 A mean-preserving spread of the income distribution always implies an increase in the Gini-coefficient (see Dalton (1920) and Cowell (2000)).

Remark 2 In order for the monopolist's profits to increase, the meanpreserving spread does not have to be such that $\bar{E}$ increases and $\underline{E}$ decreases 
for any cutoff - it suffices if this holds for the initially optimal cutoff. The proposition therefore states sufficient conditions for an increase in the monopolist's profits.

Note that the definition of a general mean-preserving spread of a distribution requires that mass from the middle of the distribution is transferred to the tails in such a way that the mean of the distribution remains constant (see Rothschild and Stiglitz (1970) or Atkinson (1970)). Formally, we say that $G(y)$ is a mean-preserving spread of $F(y)$ if $(1) \int d G(y)=\int d F(y)$ and (2) $\int_{0}^{\hat{y}}[F(y)-G(y)] d y \leq 0 \forall \hat{y} \in Y$ with strict inequality for some $\hat{y}$. It is immediate to see that this definition doesn't imply that $\bar{E}$ increases and $\underline{E}$ decreases for all cutoffs. For instance, suppose we take mass from the interval $[a, b]$ (where $0<a<b<E$ ) and transfer it to the interval $\left[a^{\prime}, b^{\prime}\right]$ (where $a^{\prime}<a$ and $\left.b^{\prime}<b\right)$ and do a symmetric shift of mass to the upper tail from an interval above the mean such that the mean stays constant. This transformation would qualify as a mean-preserving spread, but the conditional expectations at any cutoff below $a$ wouldn't change (or in other words, $\left.\int_{0}^{\hat{y}}[F(y)-G(y)] d y=0 \forall \hat{y}<a\right)$. We can ensure that the mean-preserving spread increases $\bar{E}$ and decreases $\underline{E}$ for any cutoff (and is therefore what I call "monotone") if we require that for all values of $y$ smaller than $E$, weight shifts downwards to lower values, and for all values of $y$ larger than $E$, weight shifts upwards to higher values. Formally, this would mean that $F(E)=G(E)$ and that $F$ and $G$ intersect only once, where $F$ cuts $G$ from below ("single-crossing"), and instead of $\int_{0}^{\hat{y}}[F(y)-G(y)] d y \leq 0 \forall \hat{y} \in Y$ we require $\int_{0}^{\hat{y}}[F(y)-G(y)] d y<0 \forall \hat{y} \in\left(0, y_{\max }\right){ }^{4}$

If the income distribution and the fixed cost are initially such that $\Pi\left(\hat{y}^{*}\right)<0$, an increase in inequality can have an effect on the monopolist's decision of whether or not to offer sorting at some $\hat{y}$, where she compares the profits from offering the sorting technology to 0 (the profits she would make if she stays inactive). An increase in inequality of the form described

\footnotetext{
${ }^{4}$ Such a mean-preserving spread can always be constructed if the initial distribution is strictly monotonic. The easiest way is to just transfer mass from the middle of the distribution to the very endpoints of it (i.e. 0 and $y_{\max }$ ) in such a way that the mean doesn't change.
} 
above, if it is large enough, will make the monopolist's profits positive, which in turn leads the monopolist to become active. As a result, society will become segregated due to an increase in inequality in the form of a mean-preserving spread of the income distribution.

Corollary 2 If society is not segregated initially, a sufficiently high increase in inequality in the form of a monotone mean-preserving spread will make it profitable for a monopolist to offer sorting.

A mean-preserving spread is not the only type of increase in inequality that increases the monopolist's profits from offering sorting. In fact, from examining the expression for the monopolist's profits, $\hat{y}^{*}\left(E-\underline{E}\left(\hat{y}^{*}\right)\right)-c$, it is straightforward to see that any increase in inequality that increases $E-\underline{E}\left(\hat{y}^{*}\right)$ for the initially optimal cutoff $\hat{y}^{*}$ will raise the monopolist's profits. In Appendix 7.1 I show that if $F$ is lognormal, an increase in the log-variance will also increase the monopolist's profits (if $\sigma$ is large enough).

Proposition 2 If income is lognormally distributed and the log-variance $\sigma$ is sufficiently large, an increase in $\sigma$ leads to an increase in the monopolist's maximal revenue from offering sorting.

Proof. See Appendix 7.1.

Remark 3 There is a 1-to-1 relationship between $\sigma$ and the Gini coefficient. An increase in $\sigma$ amounts to a median-preserving spread of the income distribution.

\subsection{Welfare}

The above section shows that an increase in inequality in the form of a mean-preserving spread increases the monopolist's profit. But what happens to welfare? Total welfare under no sorting is

$$
T W^{P}=\int y E f(y) d y=E^{2} .
$$


Total welfare with two groups and cutoff $\hat{y}$ is ${ }^{5}$

$$
\begin{gathered}
T W(\hat{y})=\underline{E}(\hat{y}) \int_{0}^{\hat{y}} y f(y) d y+\bar{E}(\hat{y}) \int_{\hat{y}}^{y_{\max }} y f(y) d y-(1-F(\hat{y})) \hat{y}(\bar{E}(\hat{y})-\underline{E}(\hat{y})) \\
=F(\hat{y})(\underline{E}(\hat{y}))^{2}+(1-F(\hat{y}))(\bar{E}(\hat{y}))^{2}-(1-F(\hat{y})) \hat{y}(\bar{E}(\hat{y})-\underline{E}(\hat{y})) \\
=F(\hat{y})(\underline{E}(\hat{y}))^{2}+(1-F(\hat{y}))(\bar{E}(\hat{y}))^{2}-\hat{y}(E-\underline{E}(\hat{y}))
\end{gathered}
$$

Levy and Razin (2015) characterize distributions for which sorting is always more efficient than no sorting, irrespective of the cutoff. They show the difference between welfare of sorting at cutoff $\hat{y}$ and welfare of no sorting can be written as

$$
T W(\hat{y})-T W^{P}=(E-\underline{E}(\hat{y}))(\bar{E}(\hat{y})-E-\hat{y})
$$

and thus two groups yield higher welfare than one group for any $\hat{y}$ iff the income distribution is such that

$$
\bar{E}(\hat{y})-E>\hat{y} \forall \hat{y}
$$

This condition is what has in reliability theory been termed the new worse than used in expectations (NWUE) property. A distribution $F$ is NWUE if condition (6) is satisfied, and new better than used in expectations (NBUE) if the opposite holds, i.e.

$$
\bar{E}(\hat{y})-E<\hat{y} \forall \hat{y}
$$

It is immediate to conclude the following:

1. If $F$ is NWUE, sorting at any cutoff is more efficient than no sorting.

2. If $F$ is NBUE, no sorting yields higher welfare than sorting at any $\hat{y}$.

\footnotetext{
${ }^{5}$ As in Levy and Razin (2015), total welfare from a particular partition takes into consideration the sorting fee paid (as a deadweight loss to society, or benefitting only a negligible proportion of society). If the sorting fee would not be considered, perfect sorting would always be efficient, because the utility from a match is supermodular (see Becker (1974)).
} 
3. If $F$ is not NBUE, then there will always exist some cutoff $\hat{y}$ at which sorting yields a higher welfare than no sorting.

Unless $F$ is NBUE, sorting at some cutoff $\hat{y}$ always yields higher welfare than no sorting. For instance, the lognormal distribution is not NBUE (for no parameter values), hence there always exists a cutoff $\hat{y}$ at which sorting is more efficient than no sorting. On the other hand, the uniform distribution is NBUE, hence no sorting yields higher welfare than sorting at any cutoff.

It is immediate to show that the same mean-preserving spread that increases the monopolist's profits also increases welfare at certain cutoffs $\hat{y}$.

Proposition 3 A monotone mean-preserving spread of the income distribution increases welfare from sorting at those cutoffs where $\bar{E}(\hat{y})-E>\hat{y}$.

Proof. If $\bar{E}(\hat{y})-E>\hat{y}$ then (5) tells us that the difference between welfare of sorting at $\hat{y}$ and welfare of no sorting increases due to this meanpreserving spread (both $E-\underline{E}(\hat{y})$ and $\bar{E}(\hat{y})-E-\hat{y}$ increase). As welfare of no sorting is $E^{2}$ and thus doesn't change due to a mean-preserving spread, this implies that welfare of sorting at $\hat{y}$ must increase.

Note that no general predictions can be made for welfare at those cutoffs where $\bar{E}(\hat{y})-E<\hat{y}$ : On the one hand, $E-\underline{E}(\hat{y})$ increases, but on the other hand $\bar{E}(\hat{y})-E-\hat{y}$ is negative (even though the mean-preserving spread will decrease this term in absolute value). The total effect of the mean-preserving spread on (5) is thus ambiguous and will depend on the shape of the analyzed income distribution.

If $F$ is NBUE and hence there $i s$ no cutoff such that $\bar{E}(\hat{y})-E>\hat{y}$, a mean-preserving spread can make sorting efficient for some cutoffs.

Proposition 4 If $F$ is initially NBUE, a sufficiently large monotone meanpreserving spread of the income distribution will make sorting efficient at some cutoff $\hat{y}$.

Proof. The mean-preserving spread will increase $\bar{E}(\hat{y})-E$ for all $\hat{y}$, which will eventually make $\bar{E}(\hat{y})-E-\hat{y}$ positive for some $\hat{y}$.

An increase in inequality will therefore increase welfare of sorting at those cutoffs for which $\bar{E}(\hat{y})-E-\hat{y}>0$ and can make sorting at some cutoff 
efficient if $F$ is initially NBUE. Importantly, though, it is not necessarily the case that sorting at the cutoff that the monopolist chooses after the increase in inequality yields higher welfare than before As described above, a meanpreserving spread of the income distribution increases welfare of sorting at those cutoffs for which $\bar{E}(\hat{y})-E-\hat{y}>0$, but what happens to welfare of sorting at the other cutoffs depends on the shape of the income distribution. Furthermore, even if the monopolist's optimal cutoff is initially such that $\bar{E}(\hat{y})-E-\hat{y}>0$, the change in the shape of the income distribution can imply that the monopolist chooses a different cutoff after the meanpreserving spread, at which welfare is lower than before.

The relationship between the monopolist's profit and welfare at the monopolist's optimally chosen cutoff will be the focus of the next section.

\section{Increasing inequality and the conflict be- tween monopolist profit and welfare}

The above analysis shows that an increase in inequality in the form of a monotone mean-preserving spread increases both the monopolist's profit and total welfare from sorting at some cutoffs $\hat{y}$. However, the cutoffs at which the monopolist's profit increases do not have to be the same as the ones where welfare increases. Indeed, if the monopolist chooses to offer sorting at some cutoff due to an increase in inequality, welfare from sorting at this cutoff is not necessarily higher than before - a monopolist's and a benevolent planner's interests are in general not aligned. As I will demonstrate below, total welfare of sorting at the monopolist's optimal cutoff can indeed decline with inequality. In order to show this, I will first analyze how the monopolist's optimal decision (i.e. her optimal cutoff $\left.\hat{y}^{*}\right)$ is affected by an increase in inequality, for a broad class of income distributions.

At first I will use a simple income distribution to illustrate the potential conflict between monopolist profits and welfare due to increasing inequality. I call this distribution the symmetric atom distribution. ${ }^{6}$

\footnotetext{
${ }^{6}$ This distribution, and also some of the distributions analyzed later in this paper don't satisfy all the conditions that I require in the initial setup of the model, i.e. $F$ is in general not continuous and strictly monotonic. However, this is not a problem for
} 


\subsection{Symmetric atom distribution}

Suppose $F$ has two atoms at 0 and $y_{\max }$, each with mass $z$, and is uniformly distributed in between. ${ }^{7}$ Then average income is $E(y)=\frac{y_{\max }}{2}$ and the conditional expectations are

$$
\underline{E}(\hat{y})=\frac{\left(\frac{1-2 z}{y_{\max }}\right) \frac{\hat{y}^{2}}{2}}{z+\left(\frac{1-2 z}{y_{\max }}\right) \hat{y}}
$$

and

$$
\bar{E}(\hat{y})=\frac{z y_{\max }+\left(\frac{1-2 z}{y_{\max }}\right)\left(\frac{y_{\max }^{2}}{2}-\frac{\hat{y}^{2}}{2}\right)}{z+\left(\frac{1-2 z}{y_{\max }}\right)\left(y_{\max }-\hat{y}\right)} .
$$

Note that $z$ must be in the interval $[0,0.5]$ and that $z=0$ implies that $F$ is uniformly distributed. Furthermore, $z$ parameterizes inequality (in the sense of the difference $\bar{E}-\underline{E}$ for any cutoff), and an increase in $z$ is a monotone mean-preserving spread of the income distribution (and therefore implies an increase in the Gini-coefficient of the distribution).

From Proposition 1 we know that the monopolist's profit is increasing in $z$. In order to identify how the monopolist's optimal cutoff is affected by an increase in inequality, I derive the following Lemma:

Lemma 1 If the income distribution is such that it can be written as $F(y, z)$, where $z$ parameterizes inequality and an increase in $z$ is a monotone mean-preserving spread of the income distribution, then an increase in $z$ increases the monopolist's profit-maximizing cutoff if the income distribution is such that

$$
\frac{\partial^{2} \underline{E}\left(\hat{y}^{*}, z\right)}{\partial \hat{y} \partial z} \leq 0 \quad \text { and } \quad \frac{\partial^{2} \underline{E}\left(\hat{y}^{*}, z\right)}{(\partial \hat{y})^{2}} \geq 0 .
$$

Proof. If the monopolist's maximization problem has an interior solution,

the below calculations.

${ }^{7}$ This distribution is very simple and of course not usually encountered in real-life economics. However, I use it because it is easy to handle and - despite its stylized shape - can be deployed to analyze the implications of a society that is "drifting apart", where the rich are getting richer and the poor are becoming poorer. 
the monopolist's optimal cutoff is characterized via the first order condition

$$
\frac{d R\left(\hat{y}^{*}, z\right)}{d \hat{y}}=0
$$

The monopolist's revenue is

$$
R(\hat{y}, z)=\hat{y}(E-\underline{E}(\hat{y}, z))
$$

and the optimal cutoff is thus given by

$$
E-\underline{E}\left(\hat{y}^{*}, z\right)=\hat{y}^{*} \frac{\partial \underline{E}\left(\hat{y}^{*}, z\right)}{\partial \hat{y}} .
$$

Taking the derivative with respect to $z$ gives

$$
-\frac{\partial \underline{E}\left(\hat{y}^{*}, z\right)}{\partial \hat{y}} \frac{d \hat{y}^{*}}{d z}-\frac{\partial \underline{E}\left(\hat{y}^{*}, z\right)}{\partial z}=\hat{y} \frac{\partial^{2} \underline{E}\left(\hat{y}^{*}, z\right)}{(\partial \hat{y})^{2}} \frac{d \hat{y}^{*}}{d z}+\frac{\partial \underline{E}\left(\hat{y}^{*}, z\right)}{\partial \hat{y}} \frac{d \hat{y}^{*}}{d z}+\hat{y} \frac{\partial^{2} \underline{E}\left(\hat{y}^{*}, z\right)}{\partial \hat{y} \partial z}
$$

and therefore

$$
\frac{-\frac{\partial \underline{E}\left(\hat{y}^{*}, z\right)}{\partial z}-\hat{y}^{*} \frac{\partial^{2} \underline{E}\left(\hat{y}^{*}, z\right)}{\partial \hat{y} \partial z}}{\hat{y}^{*} \frac{\partial^{2} E\left(\hat{y}^{*}, z\right)}{(\partial \hat{y})^{2}}+2 \frac{\partial \underline{\underline{y}}\left(\hat{y}^{*}, z\right)}{\partial \hat{y}}}=\frac{d \hat{y}^{*}}{d z} .
$$

Because an increase in $z$ is a monotone mean-preserving spread, we have that $\frac{\partial \underline{E}(\hat{y}, z)}{\partial z}<0$. Furthermore, an increase in the cutoff always increases average income below the cutoff, therefore $\frac{\partial \underline{E}(\hat{y}, z)}{\partial \hat{y}}>0$. Sufficient conditions for

$$
\frac{d \hat{y}^{*}}{d z}>0
$$

are therefore

$$
\frac{\partial^{2} \underline{E}\left(\hat{y}^{*}, z\right)}{\partial \hat{y} \partial z} \leq 0 \quad \text { and } \quad \frac{\partial^{2} \underline{E}\left(\hat{y}^{*}, z\right)}{(\partial \hat{y})^{2}} \geq 0
$$

The monopolist's profit maximization problem is guaranteed to have an interior solution if the revenue function is strictly concave in $\hat{y}$, i.e. $\frac{\partial^{2} R(\hat{y}, z)}{(\partial \hat{y})^{2}}<0$ for all $\hat{y}$. We have that

$$
\frac{\partial^{2} R(\hat{y}, z)}{(\partial \hat{y})^{2}}=-2 \frac{\partial \underline{E}(\hat{y}, z)}{\partial \hat{y}}-\hat{y} \frac{\partial^{2} \underline{E}(\hat{y}, z)}{(\partial \hat{y})^{2}}
$$

$\frac{\partial \underline{E}(\hat{y}, z)}{\partial \hat{y}}$ is always positive, hence the whole expression is negative for sure if 
$\frac{\partial^{2} \underline{E}(\hat{y}, z)}{(\partial \hat{y})^{2}} \geq 0$, which is exactly one of the sufficient conditions above. Hence, this condition ensures both that the monopolist's optimal cutoff is interior and (together with the condition for the cross derivative) that this optimal cutoff increases with inequality.

It is straightforward to show that the sufficient conditions from Lemma 1 hold for the symmetric atom distribution, and hence the monopolist's optimal cutoff is increasing in $z$.

Proposition 5 The monopolist's optimal cutoff is increasing in z. For $z=$ 0 the optimal cutoff is at $\frac{y_{\max }}{2}$. Hence, the monopolist's optimal cutoff is located in the interval $\left[\frac{y_{\max }}{2}, y_{\max }\right]$ for all $z$.

Proof. See Appendix 7.2.

Total welfare without sorting is independent of inequality, it is $E^{2}=$ $\frac{y_{\max }^{2}}{4}$ for all $z$. I find that for strictly positive $z$, sorting at small but positive $\hat{y}$ yields higher welfare than no sorting, but sorting at the monopolist's optimal cutoff (which, as Proposition 5 shows, is always greater than $\frac{y_{\max }}{2}$ ) is always less efficient than no sorting. Total welfare is always highest at $\hat{y}=0$, i.e. if everybody except the mass of people with 0 income is in the rich group.

Proposition 6 1. If $z=0$ (uniform distribution), maximal total welfare is achieved with no sorting.

2. If $z>0$, maximum welfare is attained at $\hat{y}=0$ for all $z$, i.e. it is optimal for the rich group to consist of everybody except people with 0 income. Furthermore, welfare of sorting at $\hat{y}=0$ is increasing in $z$.

3. If $z>0$, there is a range of $\hat{y} \geq 0$ for which sorting at these $\hat{y}$ yields higher welfare than no sorting. This range increases with $z$ and becomes $\left[0, \frac{y_{\max }}{2}\right)$ if $z=0.5$. No sorting is therefore always more efficient than sorting at the monopolist's optimal cutoff (which is always above $\left.\frac{y_{\max }}{2}\right)$.

Proof. See Appendix 7.2.

For the symmetric atom distribution, there exists a conflict between welfare and profit maximization, in the sense that no sorting is always more 
efficient than sorting at the monopolist's optimal cutoff. The following Proposition shows that this conflict increases with inequality:

Proposition 7 Welfare at the monopolist's optimum is decreasing in $z$ if $z$ is large enough.

Proof. See Appendix 7.2.

In addition to analyzing total welfare, I will also examine how welfare of the richest varies with $z$. The reason why this is interesting is that it gives us an upper bound on how much anybody in society benefits from sorting at some $\hat{y}$ compared to no sorting, due to the following Proposition:

Proposition 8 The utility difference between sorting at some cutoff $\hat{y}$ and no sorting is increasing in $y$, i.e. if a person with income y prefers no sorting to sorting at some $\hat{y}$, then also everybody with income smaller than $y$ prefers no sorting to sorting.

Proof. Utility from sorting at $\hat{y}$ for a person with income $y \geq \hat{y}$ is

$$
y \bar{E}(\hat{y})-\hat{y}(\bar{E}(\hat{y})-\underline{E}(\hat{y}))
$$

and utility from no sorting is

$$
y E
$$

hence the utility difference amounts to

$$
y \bar{E}(\hat{y})-\hat{y}(\bar{E}(\hat{y})-\underline{E}(\hat{y}))-y E=(\bar{E}(\hat{y})-\underline{E}(\hat{y}))(y F(\hat{y})-\hat{y}),
$$

where a positive difference implies that sorting at $\hat{y}$ yields higher utility than no sorting. The derivative of this difference with respect to $y$ (for given $\hat{y})$ is $F(\bar{E}(\hat{y})-\underline{E}(\hat{y}))$ which is always positive. Hence, utility of sorting is increasing in income for members of the rich group. The people just at $\hat{y}$ - who are in the rich group - will derive utility $\hat{y} \underline{E}$ and everybody in the poor group will derive less utility and it is straightforward to see that utility in the poor group is also increasing in income. Hence, utility from sorting at cutoff $\hat{y}$ is increasing in income for everybody in the economy.

I find the following results for welfare of people with income $y_{\max }$ (which I denote by $\left.W_{y_{\max }}\right)$ : 
Proposition 9 1. If $z=0$, welfare of people with income $y_{\max }$ is constant and equal to $\frac{y_{\max }^{2}}{2}$, irrespective of whether there is sorting or not.

2. If $z>0$ then welfare of people with income $y_{\max }$ is equal to $\frac{y_{\max }^{2}}{2}$ without sorting, but it is higher than $\frac{y_{\max }^{2}}{2}$ if there is sorting at any cutoff $\hat{y} \in\left[0, \frac{y_{\max }}{2}\right)$. Hence, people with income $y_{\max }$ prefer sorting at any $\hat{y} \in\left[0, \frac{y_{\max }}{2}\right)$ to no sorting. However, no sorting is always preferred to sorting at $\hat{y}>\frac{y_{\max }}{2}$.

3. $W_{y_{\max }}$ at those $\hat{y}$ for which sorting is better than no sorting (i.e. all $\hat{y}<\frac{y_{\max }}{2}$ ) increases with $z$ and is highest if $z=0.5$.

4. If $z>0, W_{y_{\max }}$ is maximized at $\hat{y}=0$, i.e. when everybody except people with zero income is in the rich group. However, no sorting is always preferred to the monopolist's optimal cutoff for $z>0$ (because the monopolist's optimal cutoff is always larger than $\left.\frac{y_{\max }}{2}\right)$.

Proof. See Appendix 7.2.

Proposition 10 Welfare of the richest from sorting at the monopolist's optimum is decreasing in $z$.

Proof. See Appendix 7.2.

As inequality increases, welfare of the richest in society from sorting at the monopolist's optimal cutoff goes down. An increase in inequality has two effects on the richest people in society: Their group gets richer on average (because there is more mass at the top end and because the cutoff increases) but at the same time they have to pay a higher sorting fee, because the difference between rich and poor, which determines the sorting fee, increases. The net effect on their welfare is negative.

Finally, in addition to looking at the richest in society, I also analyze how an increase in inequality affects welfare of sorting at the monopolist's optimal cutoff for the poor group. Here, I find the following:

Proposition 11 Average welfare in the poor group from sorting at the monopolist's optimal cutoff decreases due to an increase in inequality. 
Proof. See Appendix 7.2.

An increase in inequality has two effects on average welfare in the poor group: We know that the monopolist's optimal cutoff increases due to a rise in inequality, which benefits the poor group because people with higher incomes become members of their group and push average income up. However, this increase in the cutoff is not enough to counteract the negative effect of an increasing mass of poor people with zero income in their group, which pulls average income and average welfare down. The overall effect of an increase in inequality is thus negative.

\subsection{Generalizations}

We have seen for the case of the atom distribution that (unless $z$ is very small) welfare at the monopolist's optimal cutoff is decreasing in inequality, and that both welfare of the richest in society and average welfare in the poor group decline as well. Now I want to examine which of these findings apply to a more general class of distributions. First, I will analyze five stylized types of income distributions with the same average income that differ in their implied degree of inequality (measured as $\bar{E}-\underline{E}$ for any cutoff) and analyze how these different degrees of inequality are reflected in monopolist profits and resulting net welfare. These stylized income distributions range from total equality (where everybody in society has the same income) to a distribution that I call "high inequality" (where half of the population have nothing, and half have the maximum possible income). The examined distributions are ordered according to inequality (from most equal to least equal).

\section{- Total equality}

If the income distribution is one of total equality, i.e. where everybody has income $\frac{y_{\max }}{2}$, then the monopolist's profits will be 0 (because offering sorting will not be profitable with fixed costs or yield a profit of 0 without fixed costs). Total (net) welfare in this case is $\left(\frac{y_{\max }}{2}\right)^{2}=\frac{\left(y_{\max }\right)^{2}}{4}$. Note that total welfare without sorting only depends on the expected value of the income distribution. As average income is the same for all the distributions in this analysis, total welfare without sorting doesn't change, it is $\frac{\left(y_{\max }\right)^{2}}{4}$ in all cases. 


\section{- Triangle distribution}

If income is distributed in a triangular (isosceles) shape on $\left[0, y_{\max }\right]$ such that the density is

$$
\begin{aligned}
& f(y)=\frac{4}{\left(y_{\max }\right)^{2}} y \quad \text { if } y \in\left[0, \frac{y_{\max }}{2}\right] \\
& f(y)=\frac{4}{y_{\max }}-\frac{4}{\left(y_{\max }\right)^{2}} y \quad \text { if } y \in\left[\frac{y_{\max }}{2}, y_{\max }\right]
\end{aligned}
$$

the profit-maximizing cutoff for the monopolist is $\hat{y}^{*}=\frac{3 y_{\max }}{8}$ and the resulting profits are $\frac{3\left(y_{\max }\right)^{2}}{32}$. Welfare from sorting at this cutoff amounts to $\frac{3059}{529} \frac{\left(y_{\max }\right)^{2}}{32}<\frac{\left(y_{\max }\right)^{2}}{4}$. Hence, welfare is maximized when there is no sorting.

\section{- Uniform distribution}

If income is uniformly distributed on $\left[0, y_{\max }\right]$, the monopolist's profit maximizing cutoff is $\hat{y}^{*}=\frac{y_{\max }}{2}$ and the resulting profit is $\frac{\left(y_{\max }\right)^{2}}{8}$. Welfare at this cutoff is $\frac{3\left(y_{\max }\right)^{2}}{16}<\frac{\left(y_{\max }\right)^{2}}{4}$. Hence, welfare is maximized with no sorting.

\section{- Reverse triangle distribution}

If income is distributed in a reverse-triangular (isosceles) shape on $\left[0, y_{\max }\right]$ such that the density is

$$
\begin{aligned}
& f(y)=\frac{2}{y_{\max }}-\frac{4}{\left(y_{\max }\right)^{2}} y \quad \text { if } y \in\left[0, \frac{y_{\max }}{2}\right] \\
& f(y)=-\frac{2}{y_{\max }}+\frac{4}{\left(y_{\max }\right)^{2}} y \quad \text { if } y \in\left[\frac{y_{\max }}{2}, y_{\max }\right]
\end{aligned}
$$

the monopolist's optimal cutoff is $\hat{y}^{*}=0.64 y_{\max }$, which yields a profit of $0.1935 y_{\max }^{2}$. Total welfare at this cutoff is $0.163 y_{\max }^{2}<\frac{\left(y_{\max }\right)^{2}}{4}$. Again, no sorting would be best for welfare.

\section{- High inequality}

If half of the population has 0 income and half of them earn $y_{\max }$, the optimal cutoff for the monopolist is $\hat{y}=y_{\max }$ with corresponding sorting fee $\frac{y_{\max }}{2}$. Note that due to the jump in $F$ at $y_{\max }(F$ is not continuous here!) the sorting fee is not uniquely determined, any 
$b \in\left(0, \frac{y_{\max }}{2}\right]$ would work, and the monopolist will choose the highest in this interval to maximize her profits (and therefore the profits will be $\left.\frac{\left(y_{\max }\right)^{2}}{2}\right)$. Welfare in this case would be 0 . Welfare would be maximized with the same partition, i.e. a poor group with zero income and a rich group with income $y_{\max }$, but with the lowest of feasible sorting fees, i.e. $b$ being just $\epsilon$ over 0 . Resulting welfare would be $\frac{y_{\max }}{2}-\frac{\epsilon}{2}$. If the mass at both endpoints is not equal, this last result holds as well, because it is always better to separate rich and poor if the sorting fee is negligible, due to the supermodularity of utility from sorting (see Becker (1974)). The monopolist's profit in that latter case is increasing in the mass of rich people relative to poor people.

From this simple analysis I can conclude the following for these five distributions:

1. As inequality increases (in terms of discrete jumps from one distribution to another), the monopolist's profits increase.

2. As inequality increases, the monopolist's optimal cutoff increases.

3. Total welfare is independent of inequality in the absence of sorting, it depends only on average income. For all the above analyzed distributions, no sorting is more efficient than sorting at any cutoff $\hat{y}$.

4. If the monopolist chooses the cutoff, then welfare is highest in the case of total equality (because the sorting fee is 0 in that case and the situation is equal to no sorting, which is optimal for all the distributions discussed above). The next highest welfare would be achieved in the uniform case, followed by the triangular and then the reverse triangular case, and the case of total inequality would be worst for welfare (given the sorting fee that the monopolist would charge). Hence if we exclude the case of total equality and start from a triangular distribution - welfare of sorting at the monopolist's optimal cutoff initially increases with inequality, but as inequality becomes too high the monopolist can claim a huge part of the gross benefits from sorting for herself and net welfare decreases. 
For the symmetric atom distribution, I found that welfare from sorting at the monopolist's cutoff was decreasing in inequality. Here, we see that if we don't only look at mean-preserving spreads of the uniform distribution, but actually allow inequality also to be smaller than for a uniform distribution, the picture is different: Welfare increases with inequality for small rates of inequality, and decreases thereafter. In Appendix 7.4, I show that this is true not only for the above discrete jumps in inequality but also if we look at continuous changes in inequality for these types of distributions. In particular, I examine a distribution that is, for low levels of inequality, shaped like a house, and then as inequality increases becomes uniform and in the end looks like a reverse house (or trough). The two extreme cases are thus the triangle distribution (low inequality) and the reverse triangle distribution (high inequality) from above. I find the same results for this continuous version of the stylized distributions above: welfare of sorting at $y^{*}$ increases in inequality for low rates of inequality, and decreases for high rates. In a sense, there is thus less of a conflict between profit maximization and welfare for low rates of inequality than for high rates. However, note that all these distributions, ranging from the triangle to the reverse triangle one and all degrees of inequality in between, are NBUE and hence no sorting yields higher welfare than sorting at any cutoff (see Appendix 7.7). For low rates of inequality, an increase in inequality increases welfare at the monopolist's optimal cutoff, but a benevolent social planner would nevertheless prefer to have no sorting at all in those cases.

In Appendix 7.5, I analyze the lognormal distribution and show that monopolist profit-maximization and welfare maximization are not necessarily opposed goals if inequality is low. However, also for this type of distribution the conflict between welfare and monopolist's profits increases for high rates of inequality.

\section{$5 \quad$ Multiple groups}

The previous sections examine how increasing inequality affects welfare and profits if the monopolist can choose one cutoff and thus offer segregation into two groups. I have shown that the interests of a profit-maximizing monopolist and a benevolent social planner are generally not aligned, and that 
the conflict between those interests increases with inequality. In the following section I compare these results to a situation where the monopolist doesn't face costs of offering segregation and can therefore offer infinitely many groups (i.e. perfect sorting) if she wants. I will demonstrate that the findings from the previous sections hold in some sense also for this more general setting: There is a way in which an increase in inequality increases the conflict between monopolist's profits and welfare (and lets the monopolist extract more surplus, if she can decide on the menu of sorting fees).

Before looking at the monopolist's optimization problem, let me first examine what is best for welfare if multiple groups are possible. Hoppe et al. (2009) show that if the income distribution is such that the coefficient of variation, which is given by

$$
C V=\frac{\sqrt{\operatorname{Var}(y)}}{E(y)}
$$

is larger than 1 then perfect sorting is better than no sorting for welfare, and if $C V \leq 1$, the opposite holds:

Proposition 12 (Hoppe et al. (2009)) Perfect sorting is more (less) efficient than no sorting iff $C V \geq(\leq) 1$.

Note that the coefficient of variation is a measure of inequality - it is high if the difference between the standard deviation and the average is high, and it increases due to a mean-preserving spread of the income distribution. Hence, another way to interpret the above Proposition is in terms of inequality: For low rates of inequality, no sorting is more efficient than perfect sorting, whereas if inequality is high, perfect sorting yields higher welfare than no sorting.

It is straightforward to show that the triangle distribution, the uniform distribution and the reverse triangle distribution discussed in the previous section and the house distribution discussed in the Appendix (which encompasses all the others) are NBUE (see Appendix 7.7). As NBUE implies that the coefficient of variation is smaller than 1 , this means that no sorting yields higher welfare than perfect sorting for these distributions. 
The symmetric atom distribution is not NBUE - indeed I have shown in the previous section that for small $\hat{y}$ sorting yields higher welfare than no sorting. However, the symmetric atom distribution has $C V \leq 1$ and therefore perfect sorting always yields lower welfare than no sorting (see Appendix 7.6).

For the lognormal distribution, the coefficient of variation can be written as

$$
C V=\sqrt{e^{\sigma^{2}}-1}
$$

Hence, the coefficient of variation of a lognormal distribution depends only on $\sigma$, not on $\mu$. This is intuitive, because the $C V$ is an inequality measure, and inequality in the lognormal distribution depends on $\sigma$, and not on $\mu$ (there is also a 1-to-1 relationship between $\sigma$ and the Gini coefficient). The coefficient of variation for the lognormal distribution is greater than 1 iff

$$
\begin{aligned}
C V & \geq 1 \Longleftrightarrow \\
\sqrt{e^{\sigma^{2}}-1} & \geq 1 \Longleftrightarrow \\
\sigma & \geq \sqrt{\ln (2)} \approx 0.83
\end{aligned}
$$

Hence perfect sorting yields higher welfare than no sorting iff $\sigma \geq 0.83$. (Note: If we calibrate $\mu$ and $\sigma$ in the lognormal distribution to match the first and second moment of the US household distribution, we get $\mu \approx 10.85$ and $\sigma \approx 0.85$.)

After characterizing the class of distributions for which perfect sorting is more efficient than no sorting, I will now analyze the monopolist's optimization problem: What is the monopolist's optimal sorting schedule if she doesn't face any costs of offering the technology? Rayo (2013) characterizes the optimal placement of regions of pooling and perfect sorting, depending on the shape of the income distribution. In the following I want to examine the implications of changing inequality on the monopolist's optimal sorting schedule and total welfare.

Rayo shows that if (and only if) the function $h(y)=y-\frac{1-F(y)}{f(y)}$ is nondecreasing everywhere, perfect sorting is the profit-maximizing sorting schedule. If there are regions of $y$ for which $h(y)$ is decreasing, perfect sorting is not optimal for the monopolist and she will want to introduce 
intervals of $y$ for which she pools everybody into one joint group. ${ }^{8}$

It is immediate to see that $h$ is always decreasing if the distribution has an increasing failure rate (IFR). Hence, if a distribution exhibits IFR, perfect sorting is optimal for the monopolist. We can therefore conclude the following:

Corollary 3 If the income distribution exhibits IFR, a monopolist and a benevolent social planner have conflicting interests: No sorting is more efficient than perfect sorting or any type of finite sorting, but the monopolist wants to implement perfect sorting to maximize her profits.

Proof. Levy and Razin (2015) show that an increasing failure rate of the distribution implies that the distribution is NBUE, which in turn implies that the coefficient of variation is smaller than 1. Hence, for distributions which exhibit IFR, no sorting yields higher welfare than any finite sorting (see Levy and Razin (2015)) and perfect sorting (see Hoppe et al. (2009)).

What happens within the class of distributions for which perfect sorting is optimal for the monopolist (note that this class contains the family of IFR distributions, which are characterized by low inequality in terms of the coefficient of variation, because $I F R \Rightarrow C V \leq 1$ ) if inequality increases in the sense of a mean-preserving spread of the income distribution? We know that welfare and monopolist profit are both

$$
\int \frac{y^{2}}{2} f(y) d y=\frac{E\left(y^{2}\right)}{2}
$$

in this case, i.e. both the monopolist and the citizens get half of the total surplus from perfect sorting (see Rayo (2013)). Hence, whenever a change to the distribution happens such that perfect sorting is still optimal for the monopolist afterwards, welfare and profits are affected in the same way, i.e. a benevolent social planner's and a monopolist's interests are aligned. For instance, look at the effects of a mean-preserving spread: The variance

\footnotetext{
${ }^{8}$ Note however that there are never two pooling intervals next to each other (i.e. pooling intervals are always maximal) and that pooling is never optimal at the top end of the distribution.
} 
increases but average income doesn't change. Because of

$$
\operatorname{Var}(y)=E\left(y^{2}\right)-(E(y))^{2}
$$

this implies that $E\left(y^{2}\right)$ must increase due to a mean-preserving spread, which means that a mean-preserving spread increases both welfare and the monopolist's profits in this case.

Proposition 13 If the income distribution is such that perfect sorting is optimal for the monopolist, welfare and monopolist profits benefit equally from an increase in inequality in the form of a mean-preserving spread.

Proof. See above.

The conflict between monopolist profits and welfare is thus not further intensified as inequality increases within the class of distributions for which perfect sorting is optimal for the monopolist: A benevolent social planner would always prefer no sorting to perfect sorting, but as inequality increases, both welfare and profits increase equally.

Importantly, the above result applies to small (infinitesimal) increases in inequality, such that perfect sorting still remains optimal for the monopolist. If the shape of the distribution changes too much, perfect sorting might no longer be the optimal sorting schedule for the monopolist. For instance, it can be shown in simulations that in case of the lognormal distribution, the function $h($.$) is everywhere increasing in y$ for small $\sigma$ (below 1 ), and hence perfect sorting is optimal for the monopolist. However, as $\sigma$ increases further, there is an increasing region of $\hat{y}$ for which $h$ is decreasing, which implies that pooling some regions of $Y$ is optimal for the monopolist.

What if the income distribution is not such that perfect sorting is optimal for the monopolist? We know that total surplus (just the sum of the utilities) is always maximized with perfect sorting, due to supermodularity of the utility function (see Becker (1974)): Pooling everybody yields a total surplus of $(E(y))^{2}$ while perfect sorting yields $E\left(y^{2}\right)$, which is always larger because $E\left(y^{2}\right)=\operatorname{Var}(y)+(E(y))^{2}$. The same holds for pooling intervals of $y$. But that implies that perfect sorting is always better for welfare than any other sorting that the monopolist would design. Why? Total surplus 
is maximized with perfect sorting, anything else must yield either the same surplus or less. With perfect sorting, citizens and the monopolist share the surplus equally. If the monopolist decides that she would rather not do perfect sorting, it means she must expect a higher surplus with another way of sorting, which must mean that the citizens get less than half of total surplus (and that total surplus might even be lower than that of perfect sorting).

Proposition 14 If the income distribution is such that the monopolist doesn't want to implement perfect sorting, a benevolent social planner would always prefer perfect sorting to the monopolist's sorting schedule. With her optimal sorting schedule, the monopolist can rake more than half of the total surplus from sorting.

Proof. See above.

To conclude, the conflict between monopolist's profit and total welfare has many facets in the case of multiple groups: If the distribution exhibits IFR (which implies that $C V \leq 1$ (low inequality)), the citizens would prefer no sorting to sorting, while the monopolist wants perfect sorting, but the conflict doesn't intensify with inequality: As inequality increases (in the form of a mean-preserving spread) but we stay within the class of distributions such that the monopolist wants perfect sorting (IFR is a sufficient condition for that), welfare and monopolists profits increase equally. If we start from a situation where perfect sorting is optimal for the monopolist and inequality increases such that the monopolist implements a different sorting schedule (and pools some intervals of $Y$ ), monopolist profits will increase by more and welfare will increase by less than if sorting would still be perfect (and total surplus is either equally high or less than under perfect sorting).

What remains unexplored is what happens to profits and total welfare if we already start from a situation where perfect sorting is not optimal for the monopolist and then see an increase in inequality. I leave this question open for future research. 


\section{Conclusion}

In this paper, I have discussed how changes in inequality affect socioeconomic segregation and resulting welfare in society. I have used a simple two-group model to show that a rise in inequality always increases profits of a monopolist who offers the sorting technology. Corresponding welfare in society, however, increases in line with profits only for small rates of inequality. As inequality becomes higher, a conflict between welfare and profits arises, and welfare decreases with inequality if the monopolist implements sorting to maximize her profits. At the end of the paper I discuss how these findings generalize if the monopolist is not restricted to offer only one cutoff. If the income distribution is such that perfect sorting is optimal for the monopolist initially, the prediction is clear: there is a conflict between welfare and profits, because no sorting would be welfare maximizing. The conflict doesn't intensify for small increases in inequality, such that perfect sorting remains optimal, but the monopolist is able to capture more than half of the total surplus if pooling for some intervals of $Y$ becomes optimal. The case where perfect sorting is not optimal for the monopolist to begin with remains to be explored in future research.

In the present paper, segregation does not affect people's beliefs: they retain perfect knowledge of the whole income distribution, despite interacting only with a select (and potentially very small) group of people. In Windsteiger (2017 and 2018) I relax this assumption and explore the impact of endogenous beliefs about society that are affected by social interactions. However, I do not model the supply side of sorting explicitly in these papers. To combine these two approaches remains a promising future avenue of research. 


\section{Appendix}

\subsection{Lognormal distribution: increase in $\sigma$}

Suppose that $F$ is lognormally distributed with parameters $\mu$ and $\sigma$. The monopolist's profits from offering sorting are

$$
\Pi=R\left(\hat{y}^{*}\right)-c=\hat{y}^{*}(\bar{E}-\underline{E})\left(1-F\left(\hat{y}^{*}\right)\right)-c,
$$

where $\hat{y}^{*}$ maximizes profits. Note that $\hat{y}^{*}$ will never be 0 because then $R(\hat{y})$ would be 0 , whereas for any $\hat{y} \in(0, \infty) R(\hat{y})>0$. However, as in the lognormal case $y_{\max }=\infty$, I need an extra condition to ensure that $\hat{y}^{*}=\infty$ is not optimal. The easiest way to ensure this is to show that $R(\hat{y}) \rightarrow 0$ if $\hat{y} \rightarrow \infty$. I find that

$\lim _{\hat{y} \rightarrow \infty} R(\hat{y})=\lim _{\hat{y} \rightarrow \infty} \hat{y}(E-\underline{E})=\lim _{\hat{y} \rightarrow \infty} \frac{E-\underline{E}}{\frac{1}{\hat{y}}}=\lim _{\hat{y} \rightarrow \infty} \frac{-\frac{f(\hat{y}-\underline{E})}{F}}{-\frac{1}{\hat{y}^{2}}}=\lim _{\hat{y} \rightarrow \infty} \frac{f \hat{y}^{2}(\hat{y}-\underline{E})}{F}=0$,

where the last step comes from the fact that the third moment of the lognormal distribution is finite, which implies that $f \hat{y}^{3} \rightarrow 0$ (and thus I have shown that $R(\hat{y}) \rightarrow 0$ if $\hat{y} \rightarrow \infty$ is satisfied for any income distribution with finite third moment and continuous pdf).

If income is lognormally distributed, $R(\hat{y})$ can be written as

$$
\begin{aligned}
R(\hat{y})= & \hat{y}(\bar{E}-\underline{E})(1-F(\hat{y}))=\hat{y}(E-\underline{E}) \\
& =\hat{y} E(y)\left(1-\frac{\Phi(a-\sigma)}{\Phi(a)}\right)
\end{aligned}
$$

where

$$
a=\frac{\ln \hat{y}-\mu}{\sigma}
$$

and

$$
E(y)=e^{\mu+\frac{\sigma^{2}}{2}}
$$

What happens to the monopolist's revenue if $\sigma$ changes? Note that in general also the profit-maximizing cutoff $\hat{y}^{*}$ will change, but let me just look at the change in $\hat{y}(E-\underline{E})$ if $\sigma$ changes but $\hat{y}$ stays the same. If the expression increases with $\sigma$ while keeping $\hat{y}$ constant, then it increases 
even more with the new optimal cutoff, hence showing that $\hat{y}(E-\underline{E})$ is increasing in $\sigma$ with constant cutoff is sufficient to show that an increase in $\sigma$ increases the monopolist's revenue.

The derivative of $R(\hat{y})$ with respect to $\sigma$ is

$$
\frac{\partial R}{\partial \sigma}=\frac{\partial}{\partial \sigma}\left[\hat{y} e^{\mu+\frac{\sigma^{2}}{2}}\left(1-\frac{\Phi(a-\sigma)}{\Phi(a)}\right)\right]
$$

Let me denote

$$
G:=1-\frac{\Phi(a-\sigma)}{\Phi(a)}
$$

then

$$
\frac{\partial}{\partial \sigma}\left[\hat{y} e^{\mu+\frac{\sigma^{2}}{2}} G\right]=\hat{y} e^{\mu+\frac{\sigma^{2}}{2}}\left[\sigma G+\frac{\partial G}{\partial \sigma}\right]
$$

Note that

$\frac{\partial G}{\partial \sigma}=-\frac{\phi(a-\sigma)\left(-\frac{a}{\sigma}-1\right) \Phi(a)+\Phi(a-\sigma) \phi(a) \frac{a}{\sigma}}{\Phi(a)^{2}}=\frac{\phi(a-\sigma)\left(1+\frac{a}{\sigma}\right)}{\Phi(a)}-\frac{\Phi(a-\sigma) \phi(a) \frac{a}{\sigma}}{\Phi(a)^{2}}$

and that (7) is positive iff

$$
\sigma G+\frac{\partial G}{\partial \sigma}>0
$$

which can be written as

$$
\begin{gathered}
\Longleftrightarrow \sigma\left(1-\frac{\Phi(a-\sigma)}{\Phi(a)}\right)+\phi(a-\sigma)\left(1+\frac{a}{\sigma}\right)\left(\frac{1}{\Phi(a)}\right)-\phi(a) \frac{a}{\sigma} \frac{\Phi(a-\sigma)}{\Phi(a)^{2}}>0 \\
\Longleftrightarrow \sigma\left(1-\frac{\Phi(a-\sigma)}{\Phi(a)}\right)+ \\
+\frac{1}{\Phi(a)}\left(\phi(a-\sigma)\left(1+\frac{a}{\sigma}\right)-\phi(a) \frac{a}{\sigma} \frac{\Phi(a-\sigma)}{\Phi(a)}\right)>0
\end{gathered}
$$

In order for this to be positive, a sufficient condition is

$$
\phi(a-\sigma)\left(1+\frac{a}{\sigma}\right)-\phi(a) \frac{a}{\sigma} \frac{\Phi(a-\sigma)}{\Phi(a)}>0
$$

(because $\left.1>\frac{\Phi(a-\sigma)}{\Phi(a)}\right)$. It is immediate to see that this always holds if $a<0$ and $1+\frac{a}{\sigma} \geq 0$ (i.e. $\sigma$ large enough for a given $\hat{y}$ ). It also always holds if 
$a \geq 0$, because

$$
\begin{gathered}
\phi(a-\sigma)\left(1+\frac{a}{\sigma}\right)-\phi(a) \frac{a}{\sigma} \frac{\Phi(a-\sigma)}{\Phi(a)}>0 \\
\Longleftrightarrow \frac{\phi(a-\sigma)}{\Phi(a-\sigma)}\left(1+\frac{a}{\sigma}\right)>\frac{\phi(a)}{\Phi(a)} \frac{a}{\sigma}
\end{gathered}
$$

This last expression holds for all $a \geq 0$ because $\frac{\phi(x)}{\Phi(x)}$, which is density over distribution of a standard normal distribution, is decreasing in $x$.

Hence, the revenue from offering a cutoff at $\hat{y}$ is increasing in $\sigma$ whenever $\hat{y} \geq e^{\mu}$ or $e^{\mu}>\hat{y} \geq e^{\mu-\sigma^{2}}$ (this is what $a<0$ and $1+\frac{a}{\sigma} \geq 0$ translates to).

Remember, technically I do not need to show that the revenue increases for any $\hat{y}$, just for the optimal $\hat{y}^{*}$. If $\sigma$ is large enough then for a given $\hat{y}$, $\hat{y} \geq e^{\mu-\sigma^{2}}$ will always be satisfied. Therefore, the last remaining step to show that the monopolist's maximized revenue is increasing in $\sigma$ for large enough $\sigma$ is to show that $\hat{y}^{*}$ doesn't converge to 0 if $\sigma$ increases: then there will always exist a $\bar{\sigma}$ such that if inequality is higher than $\bar{\sigma}$, the revenue from offering a cutoff $\hat{y}$ (optimally) increases if inequality increases. To show this last step, I look at the first derivative of the revenue function

$$
R^{\prime}(\hat{y})=E(y)\left(1-\frac{\Phi(a-\sigma)}{\Phi(a)}-\frac{1}{\sigma}\left(\frac{\phi(a-\sigma)}{\Phi(a)}-\frac{\Phi(a-\sigma) \phi(a)}{(\Phi(a))^{2}}\right)\right)
$$

and determine the limit as $\sigma \rightarrow \infty$. Note that this implies that $a \rightarrow 0$ and hence $\Phi(a-\sigma) \rightarrow 0, \Phi(a) \rightarrow 0.5, \phi(a-\sigma) \rightarrow 0$ and $\phi(a) \rightarrow \phi(0)$. Hence, the expression in brackets goes to 1 , and because $E(y) \rightarrow \infty$, I get that $R^{\prime}(\hat{y}) \rightarrow \infty$. But this implies that $\hat{y}^{*}$ cannot go to zero if $\sigma \rightarrow \infty$, because this means that at any cutoff $\hat{y}$, the gain from increasing it a little bit becomes infinitely large.

Hence, if $\sigma$ is large enough the profit from offering segregation is sure to increase with $\sigma$, i.e. (7) is $>0$ for sure. This means that if inequality is already high and increases further, this will increase the monopolist's revenue. 


\subsection{Proofs for the symmetric atom distribution}

Proposition 15 The monopolist's optimal cutoff is increasing in z. For $z=0$ the optimal cutoff is at $\frac{y_{\max }}{2}$. Hence, the monopolist's optimal cutoff is located in the interval $\left[\frac{y_{\max }}{2}, y_{\max }\right]$ for all $z$.

Proof. As Lemma 1 establishes, sufficient conditions for

$$
\frac{d \hat{y}^{*}}{d z}>0
$$

are

$$
\frac{\partial^{2} \underline{E}\left(\hat{y}^{*}, z\right)}{\partial \hat{y} \partial z}<0 \quad \text { and } \quad \frac{\partial^{2} \underline{E}\left(\hat{y}^{*}, z\right)}{(\partial \hat{y})^{2}}>0 .
$$

Show that $\frac{\partial^{2} \underline{E}(\hat{y}, z)}{\partial \hat{y} \partial z}<0$ : (Note: To simplify the notation I set $y_{\max }=1$ in the following calculations, but everything works analogously if the distribution is scaled up to a general $y_{\max }>0$ ).

$$
\begin{aligned}
& \underline{E}(\hat{y}, z)=\frac{(1-2 z) \frac{\hat{y}^{2}}{2}}{z+(1-2 z) \hat{y}} \\
& \frac{\partial \underline{E}(\hat{y}, z)}{\partial \hat{y}}=\frac{(1-2 z) \hat{y}(z+(1-2 z) \hat{y})-(1-2 z)^{2} \frac{\hat{y}^{2}}{2}}{(z+(1-2 z) \hat{y})^{2}} \\
& =\frac{(1-2 z)}{z+(1-2 z) \hat{y}}\left(\hat{y}-\frac{(1-2 z) \frac{\hat{y}^{2}}{2}}{z+(1-2 z) \hat{y}}\right) \\
& \frac{\partial^{2} \underline{E}(\hat{y}, z)}{\partial \hat{y} \partial z}=\frac{-2(z+(1-2 z) \hat{y}-(1-2 z)(1-2 \hat{y})}{(z+(1-2 z) \hat{y})^{2}}\left(\hat{y}-\frac{(1-2 z) \frac{\hat{y}^{2}}{2}}{z+(1-2 z) \hat{y}}\right) \\
& -\frac{(1-2 z)}{z+(1-2 z) \hat{y}}\left(\frac{\hat{y}^{2}}{2}\left(\frac{-2(z+(1-2 z) \hat{y})-(1-2 z)(1-2 \hat{y})}{(z+(1-2 z) \hat{y})^{2}}\right)\right) \\
& =\frac{-1}{(z+(1-2 z) \hat{y})^{2}}\left(\hat{y}-\frac{(1-2 z) \hat{y}^{2}}{z+(1-2 z) \hat{y}}\right) \\
& =\frac{-1}{(z+(1-2 z) \hat{y})^{2}}\left(\frac{z+(1-2 z) \hat{y}-(1-2 z) \hat{y}}{z+(1-2 z) \hat{y}}\right) \hat{y} \\
& =\frac{-1}{(z+(1-2 z) \hat{y})^{2}}\left(\frac{z}{z+(1-2 z) \hat{y}}\right) \hat{y}<0
\end{aligned}
$$


Show that $\frac{\partial^{2} \underline{E}(\hat{y}, z)}{(\partial \hat{y})^{2}}>0$ :

$$
\begin{aligned}
& \frac{\partial^{2} \underline{E}(\hat{y}, z)}{(\partial \hat{y})^{2}}=-\frac{(1-2 z)^{2}}{(z+(1-2 z) \hat{y})^{2}}\left(\hat{y}-\frac{(1-2 z) \frac{\hat{y}^{2}}{2}}{z+(1-2 z) \hat{y}}\right) \\
&+\frac{(1-2 z)}{z+(1-2 z) \hat{y}}\left(1+\frac{(1-2 z)^{2}}{(z+(1-2 z) \hat{y})^{2}} \frac{\hat{y}^{2}}{2}-\frac{(1-2 z) \hat{y}}{z+(1-2 z) \hat{y}}\right) \\
&=\frac{(1-2 z)}{z+(1-2 z) \hat{y}}\left(1-\frac{(1-2 z) \hat{y}}{z+(1-2 z) \hat{y}}\right)^{2}=\frac{(1-2 z)}{z+(1-2 z) \hat{y}}\left(\frac{z}{z+(1-2 z) \hat{y}}\right)^{2}>0
\end{aligned}
$$

If $z=0$, then

$$
\underline{E}(\hat{y})=\frac{\hat{y}}{2}
$$

and therefore

$$
R(\hat{y})=\hat{y}\left(\frac{y_{\max }}{2}-\frac{\hat{y}}{2}\right)=\frac{\hat{y} y_{\max }}{2}-\frac{\hat{y}^{2}}{2}
$$

This implies that profit is maximized at $\frac{y_{\max }}{2}$ for $z=0$.

Proposition 16 1. If $z=0$ (uniform distribution), maximal total welfare is achieved with no sorting.

2. If $z>0$, maximum welfare is attained at $\hat{y}=0$ for all $z$, i.e. it is optimal for the rich group to consist of everybody except people with 0 income. Furthermore, welfare of sorting at $\hat{y}=0$ is increasing in $z$.

3. If $z>0$, there is a range of $\hat{y} \geq 0$ for which sorting at these $\hat{y}$ yields higher welfare than no sorting. This range increases with $z$ and becomes $\left[0, \frac{y_{\max }}{2}\right)$ if $z=0.5$. No sorting is therefore always more efficient than sorting at the monopolist's optimal cutoff (which is always above $\left.\frac{y_{\max }}{2}\right)$.

Proof. First of all, note that for strictly positive $z$ welfare jumps at 0 : If people with 0 income are included in the group (so there is only one group), total welfare is $\frac{y_{\max }^{2}}{4}$, if 0 is excluded, then welfare is $\frac{y_{\max }^{2}}{4-4 z}>\frac{y_{\max }^{2}}{4}$.

For the remainder of this proof I will again set $y_{\max }=1$ for simplicity 
of notation. Total welfare at cutoff $\hat{y}$ is then given by

$$
\begin{aligned}
T W(\hat{y})= & (z+(1-2 z) \hat{y})\left(\frac{(1-2 z) \frac{\hat{y}^{2}}{2}}{(z+(1-2 z) \hat{y})}\right)^{2} \\
& +(z+(1-2 z)(1-\hat{y}))\left(\frac{z+(1-2 z)\left(\frac{1}{2}-\frac{\hat{y}^{2}}{2}\right)}{z+(1-2 z)(1-\hat{y})}\right)^{2} \\
& -\hat{y}\left(\frac{1}{2}-\frac{(1-2 z) \frac{\hat{y}^{2}}{2}}{z+(1-2 z) \hat{y})}\right)
\end{aligned}
$$

If $z=0$ the distribution becomes uniform and total welfare is

$$
T W_{z=0}(\hat{y})=\hat{y} \frac{\hat{y}^{2}}{4}+(1-\hat{y})\left(\frac{\frac{1}{2}\left(1-\hat{y}^{2}\right)}{(1-\hat{y})}\right)^{2}-\hat{y}\left(\frac{1}{2}-\frac{\hat{y}}{2}\right)=\frac{1}{4}-\frac{\hat{y}}{4}+\frac{\hat{y}^{2}}{4}
$$

It is straightforward to see that this quadratic function reaches its minimum at $\hat{y}=0.5$ and is maximized at the endpoints of the examined interval $Y$, i.e. $\hat{y}=0$ and $\hat{y}=1$, where total welfare is $\frac{1}{4}$, which is equal to the total welfare of no sorting. Hence, no sorting is (weakly) preferred to sorting at any cutoff $\hat{y} \in Y$ if $z=0$.

For the general case, where $z \neq 0$, note first that total welfare at cutoff 0 is

$$
T W(0)=\frac{\left(z+(1-2 z) \frac{1}{2}\right)^{2}}{z+(1-2 z)}=\frac{1}{4(1-z)}
$$

which is increasing in $z$ for all $z \in[0,0.5]$. It is also straightforward to see that this expression is always larger than $\frac{1}{4}$ (welfare of no sorting) if $z>0$.

At cutoff 1 this becomes

$$
T W(1)=\frac{1-2 z}{4(1-z)}
$$

which is decreasing in $z$ for all $z \in[0,0.5]$. Note that this is always smaller than $\frac{1}{4}$ (welfare of no sorting) for all $z>0$.

For all cutoffs in between 0 and 1 , note that from the previous section we know that sorting yields higher welfare than no sorting at cutoff $\hat{y}$ iff

$$
\bar{E}-E-\hat{y}>0
$$


Plugging in the expressions for $\bar{E}$ and $E$ for the atom distribution, this condition becomes

$$
\frac{(1-2 z) \hat{y}^{2}-\hat{y}+z}{2(z+(1-2 z)(1-\hat{y}))}>0 .
$$

As the numerator of this fraction is positive for all $z$ and $\hat{y}$, the condition can be simplified to

$$
(1-2 z) \hat{y}^{2}-\hat{y}+z>0
$$

It is immediate to see that this condition never holds if $z=0$, holds for all $z>0$ at cutoff 0 , and holds for all $\hat{y} \leq 0.5$ if $z=0.5$. The roots of $(1-2 z) \hat{y}^{2}-\hat{y}+z$ are

$$
y_{1,2}=\frac{1 \pm \sqrt{1-4 z+8 z^{2}}}{2-4 z}
$$

and the polynomial is positive for all $\hat{y}$ that are either smaller than the smaller of the two or larger than the larger of the two roots. As the larger root is always $\geq 1$, the only relevant case for us is the range of $\hat{y}$ smaller than $y_{1}=\frac{1-\sqrt{1-4 z+8 z^{2}}}{2-4 z}$. The value of $y_{1}$ is 0 if $z=0$ and is then increasing in $z$, until it reaches $y_{1}=0.5$ for $z=0.5$. Hence, the range of $\hat{y}$ for which sorting is better than no sorting is $\left[0, y_{1}(z)\right]$ for all $z>0$ where $y_{1}(z)$ is increasing in $z, 0$ for $z=0$ and reaches 0.5 for $z=0.5$.

Proposition 17 Welfare at the monopolist's optimum is decreasing in $z$ if $z$ is large enough.

Proof. The derivative of total welfare with respect to $z$ at the monopolist's optimal cutoff $\hat{y}^{*}$ amounts to

$$
\begin{gathered}
\frac{d T W\left(\hat{y}^{*}, z\right)}{d z}=\left(f\left(\underline{E}^{2}-\bar{E}^{2}\right)+F 2 \underline{E} \frac{\partial \underline{E}}{\partial \hat{y}}+(1-F) 2 \bar{E} \frac{\partial \bar{E}}{\partial \hat{y}}\right) \frac{d \hat{y}^{*}}{d z} \\
+\frac{\partial F}{\partial z}\left(\underline{E}^{2}-\bar{E}^{2}\right)+F 2 \underline{E} \frac{\partial \underline{E}}{\partial z}+(1-F) 2 \bar{E} \frac{\partial \bar{E}}{\partial z}-\frac{d \Pi\left(\hat{y}^{*}, z\right)}{d z}
\end{gathered}
$$

where $\Pi\left(\hat{y}^{*}, z\right)$ is the monopolist's maximized profit. We know that the monopolist's profit maximization always has an interior solution (see Lemma 1 and Proposition 5). Hence the optimal cutoff $y^{*}$ is characterized via the first order condition

$$
\frac{\partial \Pi\left(\hat{y}^{*}, z\right)}{\partial \hat{y}}=0
$$


This implies that

$$
\frac{d \Pi\left(\hat{y}^{*}, z\right)}{d z}=\frac{\partial \Pi\left(\hat{y}^{*}, z\right)}{\partial \hat{y}} \frac{d \hat{y}^{*}}{d z}-\hat{y}^{*} \frac{\partial \underline{E}}{\partial z}=-\hat{y}^{*} \frac{\partial \underline{E}}{\partial z}(>0) .
$$

Hence, the above expression can be simplified to

$$
\begin{gathered}
\frac{d T W\left(\hat{y}^{*}, z\right)}{d z}=f(\bar{E}-\underline{E})\left(\bar{E}+\underline{E}-2 \hat{y}^{*}\right) \frac{d \hat{y}^{*}}{d z}+ \\
+\frac{\partial F}{\partial z}\left(\underline{E}^{2}-\bar{E}^{2}\right)+\left(F 2 \underline{E}+\hat{y}^{*}\right) \frac{\partial \underline{E}}{\partial z}+(1-F) 2 \bar{E} \frac{\partial \bar{E}}{\partial z}
\end{gathered}
$$

(where I also use the fact that $\frac{\partial \underline{E}(\hat{y}, z)}{\partial \hat{y}}=f(\hat{y}, z) \frac{\hat{y}-\underline{E}(\hat{y}, z)}{F(\hat{y}, z)}$ and $\frac{\partial \bar{E}(\hat{y}, z)}{\partial \hat{y}}=$ $\left.f(\hat{y}, z) \frac{\bar{E}(\hat{y}, z)-\hat{y}}{1-F(\hat{y}, z)}\right)$.

We have

$$
\frac{\partial F}{\partial z}=1-2 \hat{y}
$$

and

$$
\frac{\partial \underline{E}}{\partial z}=\frac{-\frac{\hat{y}^{2}}{2}}{(z+(1-2 z) \hat{y})^{2}}
$$

and

$$
\frac{\partial \bar{E}}{\partial z}=\frac{\frac{1}{2}(\hat{y}-1)^{2}}{(z+(1-2 z)(1-\hat{y}))^{2}}
$$

Note that $\frac{\partial E}{\partial z}<0, \frac{\partial F}{\partial z}<0$ (because $\hat{y}^{*}>\frac{y_{\max }}{2}$ ) and $\frac{\partial \bar{E}}{\partial z}>0$. From Proposition 5 we know that $\frac{d \hat{y}^{*}}{d z}>0$, hence sufficient conditions for $\frac{d T W\left(\hat{y}^{*}, z\right)}{d z}<0$ are that

$$
\bar{E}+\underline{E}-2 \hat{y}^{*}<0
$$

and

$$
\frac{\partial F}{\partial z}\left(\underline{E}^{2}-\bar{E}^{2}\right)+\left(F 2 \underline{E}+\hat{y}^{*}\right) \frac{\partial \underline{E}}{\partial z}+(1-F) 2 \bar{E} \frac{\partial \bar{E}}{\partial z}<0
$$

The first condition can easily be shown to always hold for $\hat{y}^{*}>0.5$ : Plugging in the expressions for $\bar{E}$ and $\underline{E}$ yields

$$
\bar{E}+\underline{E}-2 \hat{y}^{*}=\left(\frac{z}{2}-z \hat{y}\right)+\left(2 \hat{y}-6 \hat{y}^{2}+4 \hat{y}^{3}\right)\left(\frac{1}{4}+z^{2}-z\right)
$$

We have that

$$
\left(\frac{z}{2}-z \hat{y}\right)<0 \quad \forall \hat{y}>0.5
$$


and

$$
\left(2 \hat{y}-6 \hat{y}^{2}+4 \hat{y}^{3}\right)<0 \quad \forall \hat{y}>0.5
$$

while

$$
\left(\frac{1}{4}+z^{2}-z\right)>0 \quad \forall z>0.5
$$

Hence, the total expression is always negative for $\hat{y}>0.5$. For the second condition, note that

$$
\begin{gathered}
\frac{\partial F}{\partial z}\left(\underline{E}^{2}-\bar{E}^{2}\right)+\left(F 2 \underline{E}+\hat{y}^{*}\right) \frac{\partial \underline{E}}{\partial z}+(1-F) 2 \bar{E} \frac{\partial \bar{E}}{\partial z}= \\
=\frac{(1-2 z)^{2}\left(\frac{\hat{y}^{4}}{4}-\frac{\hat{y}^{5}}{2}\right)-(1-2 z) \frac{\hat{y}^{4}}{2}-\frac{\hat{y}^{3}}{2}}{(z+(1-2 z) \hat{y})^{2}}+ \\
+\frac{z(1-y)^{2}+(1-2 z)\left(\frac{1}{2}-\frac{\hat{y}^{2}}{2}\right)(1-\hat{y})^{2}-(1-2 \hat{y})\left(z+(1-2 z)\left(\frac{1}{2}-\frac{\hat{y}^{2}}{2}\right)\right)^{2}}{(z+(1-2 z)(1-\hat{y}))^{2}}
\end{gathered}
$$

The first summand of this expression is negative for all $\hat{y}>0.5$ and all $z$, but the second term is always positive (note that $1-2 \hat{y}<0$ for all $\hat{y}>0.5$ ). If $z=0$ the sum of the two becomes $\frac{1}{4}-\frac{1}{2} \hat{y}$ which is negative for all $\hat{y}>0.5$, however if $z>0$ then there is a small range of $\hat{y}>0.5$ for which the second term is higher in absolute value than the first and hence the whole expression is positive. Indeed it can be shown that the entire expression for $\frac{d T W\left(\hat{y}^{*}, z\right)}{d z}$ is positive for small $\hat{y}^{*}>0.5$ (from numerical simulations). As $\hat{y}^{*}$ is close to 0.5 for small $z$ this implies that total welfare from sorting at the monopolist's optimal cutoff increases with $z$ for very small $z$. However, note that the monopolist's optimal cutoff increases with $z$ as well, and this increase moves $\hat{y}^{*}$ out of the area for which total welfare increases with $z$ quickly. It can be seen (from simulations) that for all $z>0.05$ the small range of $\hat{y}$ for which total welfare increases with $z$ is below $\hat{y}^{*}$ for all $z$. Hence, total welfare from sorting at the monopolist's optimal cutoff decreases with $z$ if $z>0.05$.

Proposition 18 1. If $z=0$, welfare of people with income $y_{\max }$ is constant and equal to $\frac{y_{\max }^{2}}{2}$, irrespective of whether there is sorting or not. 
2. If $z>0$ then welfare of people with income $y_{\max }$ is equal to $\frac{y_{\max }^{2}}{2}$ without sorting, but it is higher than $\frac{y_{\max }^{2}}{2}$ if there is sorting at any cutoff $\hat{y} \in\left[0, \frac{y_{\max }}{2}\right)$. Hence, people with income $y_{\max }$ prefer sorting at any $\hat{y} \in\left[0, \frac{y_{\max }}{2}\right)$ to no sorting. However, no sorting is always preferred to sorting at $\hat{y}>\frac{y_{\max }}{2}$.

3. $W_{y_{\max }}$ at those $\hat{y}$ for which sorting is better than no sorting (i.e. all $\left.\hat{y}<\frac{y_{\max }}{2}\right)$ increases with $z$ and is highest if $z=0.5$.

4. If $z>0, W_{y_{\max }}$ is maximized at $\hat{y}=0$, i.e. when everybody except people with zero income is in the rich group. However, no sorting is always preferred to the monopolist's optimal cutoff for $z>0$ (because the monopolist's optimal cutoff is always larger than $\left.\frac{y_{\max }}{2}\right)$.

Proof. Welfare of people with income $y_{\max }$ can be calculated as

$$
W_{y_{\max }}(\hat{y}, z)=y_{\max } \bar{E}-\hat{y}(\bar{E}-\underline{E}) .
$$

This can be written as (again set $y_{\max }=1$ )

$$
W_{y_{\max }}(\hat{y}, z)=\frac{\frac{z}{2}-\frac{3}{2} z \hat{y}+\frac{z \hat{y}^{2}}{2}+z \hat{y}^{3}+z^{2} \hat{y}^{2}-2 z^{2} \hat{y}^{3}+\frac{\hat{y}}{2}-\frac{\hat{y}^{2}}{2}}{(z+(1-2 z) \hat{y})(z+(1-2 z)(1-\hat{y}))} .
$$

If $z=0$ (uniform distribution) this becomes

$$
W_{y_{\max }}(\hat{y}, 0)=\frac{1}{2}
$$

Note that utility of no sorting is also $\frac{1}{2}$ for people with income $y_{\max }$, they are therefore indifferent between sorting and no sorting at any cutoff if $z=0$.

If $z>0$ : When is $W_{y_{\max }}(\hat{y})>0.5$ (=utility from no sorting), i.e. for what range of cutoffs is sorting preferred to no sorting for the richest people?

$$
\begin{gathered}
W_{y_{\max }}(\hat{y}, z)>0.5 \\
\Longleftrightarrow \frac{z}{2}-\frac{3}{2} z \hat{y}+\frac{z \hat{y}^{2}}{2}+z \hat{y}^{3}+z^{2} \hat{y}^{2}-2 z^{2} \hat{y}^{3}+\frac{\hat{y}}{2}-\frac{\hat{y}^{2}}{2}>\frac{(z+(1-2 z) \hat{y})(z+(1-2 z)(1-\hat{y}))}{2} \\
z\left(\hat{y}-3 \hat{y}^{2}+2 \hat{y}^{3}\right)+z^{2}\left(6 \hat{y}^{2}-4 \hat{y}^{3}-4 \hat{y}+1\right)>0
\end{gathered}
$$


If $z>0$ this becomes

$$
\left(\hat{y}-3 \hat{y}^{2}+2 \hat{y}^{3}\right)>z\left(-6 \hat{y}^{2}+4 \hat{y}^{3}+4 \hat{y}-1\right)
$$

The RHS is positive if $\hat{y} \geq 0.5$, which is also exactly when the LHS is negative. (The polynomial on the left has roots 1 and 0.5 and is smaller than zero in between the two and larger than zero elsewhere. The polynomial on the right has only root 0.5 in the interval $[0,1]$ and is positive above 0.5 and negative below). In other words, the inequality cannot hold for any positive $z$ if $\hat{y} \geq 0.5$ and will always hold if $\hat{y} \leq 0.5$. This means that for any $z$ the richest people prefer sorting to no sorting at any cutoff below 0.5. It is straightforward to see that they are always indifferent between sorting and no sorting at $\hat{y}=0.5$. The maximum utility is reached at $\hat{y}=0$ (meaning that the rich group consists of everybody except the poor with zero income) for any $z>0$, which can be concluded from the fact that $\frac{d W_{y_{\max }(\hat{y}, z)}}{d \hat{y}}<0$ for all $\hat{y} \in[0,1]$.

Proof that $\frac{d W_{y_{\max }}(\hat{y}, z)}{d \hat{y}}$ for all $\hat{y} \in[0,1]$ :

$$
\frac{d W_{y_{\max }}(\hat{y}, z)}{d \hat{y}}=\frac{-z^{4}+(1-2 z) z^{3}\left(-0.5+3 \hat{y}^{2}-3 \hat{y}\right)+(1-2 z)^{2} 2 z^{2} \hat{y}(-1+\hat{y})}{+(1-2 z)^{3} z\left(-\hat{y}^{2}-\hat{y}^{4}+2 \hat{y}^{3}\right)}
$$

The denominator is always positive, so it suffices to focus on the numerator. Analysis of the factors that multiply the potencies of $(1-2 z)$ shows that they are negative for all $\hat{y} \in[0,1]$ and hence $\frac{d W_{y_{\max }(\hat{y}, z)}}{d \hat{y}}$ is smaller than 0 for all $\hat{y} \in[0,1]$. The maximum welfare for the rich is therefore achieved when $\hat{y}=0$ (i.e. the rich group consists of everybody except the poorest with income 0 ). This maximum welfare is increasing in $z$ :

$$
W_{y_{\max }}(0, z)=\frac{1}{2(1-z)}
$$

On the other hand, welfare at $\hat{y}=y_{\max }(=1)$ is decreasing in $z$ :

$$
W_{y_{\max }}(1, z)=\frac{\frac{1}{2}-z}{(1-z)} .
$$


Proposition 19 Welfare of the richest from sorting at the monopolist's optimum is decreasing in $z$.

\section{Proof.}

$$
W_{y_{\max }}\left(\hat{y}^{*}, z\right)=\left(y_{\max }-\hat{y}^{*}\right) \bar{E}\left(\hat{y}^{*}\right)+\hat{y}^{*} \underline{E}\left(\hat{y}^{*}\right)
$$

The monopolist's optimal cutoff satisfies the FOC and hence

$$
E-\underline{E}\left(\hat{y}^{*}\right)=\hat{y}^{*} \frac{\partial \underline{E}\left(\hat{y}^{*}\right)}{\partial \hat{y}}
$$

The derivative of $W_{y_{\max }}\left(\hat{y}^{*}, z\right)$ with respect to $z$ is:

$\frac{d W_{y_{\max }}\left(\hat{y}^{*}, z\right)}{d z}=\left(y_{\max }-\hat{y}^{*}\right)\left(\frac{\partial \bar{E}}{\partial z}\right)+\hat{y}^{*} \frac{\partial \underline{E}}{\partial z}+\left[\left(y_{\max }-\hat{y}^{*}\right) \frac{\partial \bar{E}}{\partial \hat{y}}-(\bar{E}-\underline{E})+\hat{y}^{*} \frac{\partial \underline{E}}{\partial \hat{y}}\right] \frac{d \hat{y}^{*}}{d z}$

Using (9) this becomes

$\frac{d W_{y_{\max }}\left(\hat{y}^{*}, z\right)}{d z}=\left(y_{\max }-\hat{y}^{*}\right)\left(\frac{\partial \bar{E}}{\partial z}\right)+\hat{y}^{*} \frac{\partial \underline{E}}{\partial z}+\left[\left(y_{\max }-\hat{y}^{*}\right) \frac{\partial \bar{E}}{\partial \hat{y}}-(\bar{E}-E)\right] \frac{d \hat{y}^{*}}{d z}$

Hence, sufficient conditions for $W_{y_{\max }}\left(\hat{y}^{*}, z\right)$ to be decreasing in $z$ are that

$$
\left(y_{\max }-\hat{y}^{*}\right)\left(\frac{\partial \bar{E}}{\partial z}\right)+\hat{y}^{*} \frac{\partial \underline{E}}{\partial z}<0
$$

and

$$
\left[\left(y_{\max }-\hat{y}^{*}\right) \frac{\partial \bar{E}}{\partial \hat{y}}-(\bar{E}-E)\right]<0
$$

(because we already know that $\frac{d \hat{y}^{*}}{d z}>0$ ). Again setting $y_{\max }=1$ and using

$$
\begin{gathered}
\frac{\partial \bar{E}}{\partial z}=\frac{\frac{1}{2}(1-\hat{y})^{2}}{(z+(1-2 z)(1-\hat{y}))^{2}} \\
\frac{\partial \underline{E}}{\partial z}=\frac{-\hat{y}^{2}}{2(z+(1-2 z) \hat{y})^{2}}
\end{gathered}
$$

and

$$
\frac{\partial \bar{E}}{\partial \hat{y}}=(1-2 z)(1-\hat{y}) \frac{z+(1-2 z)\left(\frac{1}{2}-\frac{\hat{y}}{2}\right)}{(z+(1-2 z)(1-\hat{y}))^{2}},
$$

it can be shown that both terms are negative for $\hat{y}^{*} \in[0.5,1]$ and all 
$z \in[0,0.5]:$ I get that

$$
\begin{gathered}
\left(y_{\max }-\hat{y}^{*}\right)\left(\frac{\partial \bar{E}}{\partial z}\right)+\hat{y}^{*} \frac{\partial \underline{E}}{\partial z}=\frac{(1-\hat{y})^{3}}{2(z+(1-2 z)(1-\hat{y}))^{2}}-\frac{\hat{y}^{3}}{2(z+(1-2 z) \hat{y})^{2}} \\
=\frac{(1-\hat{y})^{3}(z+(1-2 z) \hat{y})^{2}-\hat{y}^{3}(z+(1-2 z)(1-\hat{y}))^{2}}{2(z+(1-2 z)(1-\hat{y}))^{2}(z+(1-2 z) \hat{y})^{2}} .
\end{gathered}
$$

The denominator is positive, and the numerator can be simplified to give

$z^{2}\left(1-3 \hat{y}+3 \hat{y}^{2}-2 \hat{y}^{3}\right)+\left(2 z-4 z^{2}\right)\left(\hat{y}-3 \hat{y}^{2}+2 \hat{y}^{3}\right)+(1-2 z)^{2} \hat{y}^{2}\left(1-4 \hat{y}+5 \hat{y}^{2}-2 \hat{y}^{3}\right)$.

It turns out that the polynomials of $\hat{y}$ in each summand are negative for all $\hat{y} \in[0.5,1]$, hence the expression is negative for the relevant ranges of $\hat{y}$ and all $z$.

Furthermore,

$$
\begin{gathered}
{\left[\left(y_{\max }-\hat{y}^{*}\right) \frac{\partial \bar{E}}{\partial \hat{y}}-(\bar{E}-E)\right]=(1-2 z)(1-\hat{y})^{2} \frac{z+(1-2 z)\left(\frac{1}{2}-\frac{\hat{y}}{2}\right)}{(z+(1-2 z)(1-\hat{y}))^{2}}} \\
-\frac{z+(1-2 z)\left(\frac{1}{2}-\frac{\hat{y}^{2}}{2}\right)}{z+(1-2 z)(1-\hat{y})}+\frac{1}{2} \\
=\frac{-z^{2}+(1-2 z) z\left(1-4 \hat{y}+3 \hat{y}^{2}\right)+(1-2 z)^{2}\left(1-4 \hat{y}+5 \hat{y}^{2}-2 \hat{y}^{3}\right)}{2(z+(1-2 z)(1-\hat{y}))^{2}}
\end{gathered}
$$

Again the denominator is positive and all the polynomials of $\hat{y}$ in the numerator are negative $\forall \hat{y} \in[0.5,1]$, which implies that the expression is negative for the relevant ranges of $\hat{y}$ and all $z$. Hence, $\frac{d W_{y_{\max }}\left(\hat{y}^{*}, z\right)}{d z}$ is negative for all $\hat{y}$ in $[0.5,1]$ for all $z$ and thus $W_{y_{\max }}\left(\hat{y}^{*}, z\right)$ is decreasing in $z$.

Proposition 20 Average welfare in the poor group from sorting at the monopolist's optimal cutoff decreases due to an increase in inequality.

Proof. Average welfare in the poor group amounts to $\underline{E}^{2}$ (note that they don't have to pay the sorting fee $b$ ). We know that

$$
\frac{d \underline{E}\left(\hat{y}^{*}, z\right)}{d z}=\frac{\partial \underline{E}}{\partial \hat{y}} \frac{d \hat{y}^{*}}{d z}+\frac{\partial \underline{E}}{\partial z}
$$


From above we know that

$$
\frac{\partial \underline{E}}{\partial \hat{y}}=\frac{(1-2 z)\left(\hat{y} z+(1-2 z) \frac{\hat{y}^{2}}{2}\right)}{(z+(1-2 z) \hat{y})^{2}}
$$

and

$$
\frac{\partial \underline{E}}{\partial z}=\frac{-\hat{y}^{2}}{2(z+(1-2 z) \hat{y})^{2}}
$$

and plugging in all the expressions for the derivatives in $\frac{d \hat{y}^{*}}{d z}$ yields

$$
\frac{d \hat{y}^{*}}{d z}=\frac{\left(\frac{3 z \hat{y}}{2}+(1-2 z) \frac{\hat{y}^{2}}{2}\right)}{(1-2 z)\left(3 z^{2}+3(1-2 z) \hat{y} z+(1-2 z)^{2} \hat{y}^{2}\right)}
$$

Hence, after simplifications, we get that

$$
\frac{d \underline{E}\left(\hat{y}^{*}, z\right)}{d z}=\frac{-(1-2 z)^{2} \frac{\hat{y}^{4}}{4}-(1-2 z) \frac{\hat{y}^{3} z}{4}}{\left(3 z^{2}+3(1-2 z) \hat{y} z+(1-2 z)^{2} \hat{y}^{2}\right)(z+(1-2 z) \hat{y})^{2}}
$$

The denominator is always positive and the numerator is always negative, hence $\frac{d E\left(\hat{y}^{*}, z\right)}{d z}<0$.

\subsection{Calculations for Section 4.2}

\subsubsection{Triangle distribution}

If the density is

$$
\begin{aligned}
& f(y)=\frac{4}{\left(y_{\max }\right)^{2}} y \quad \text { if } y \in\left[0, \frac{y_{\max }}{2}\right] \\
& f(y)=\frac{4}{y_{\max }}-\frac{4}{\left(y_{\max }\right)^{2}} y \quad \text { if } y \in\left[\frac{y_{\max }}{2}, y_{\max }\right]
\end{aligned}
$$

then I can calculate that if the cutoff $\hat{y}$ is in $\left[0, \frac{y_{\max }}{2}\right]$ the conditional expectations are

$$
\underline{E}(\hat{y})=\frac{2 \hat{y}}{3}
$$

and

$$
\bar{E}(\hat{y})=\frac{\frac{y_{\max }}{2}-\frac{4 \hat{y}^{3}}{3 y_{\max }^{2}}}{1-\frac{2 \hat{y}^{2}}{y_{\max }^{2}}}
$$


whereas if $\hat{y} \in\left[\frac{y_{\max }}{2}, y_{\max }\right]$ the expressions become

$$
\underline{E}(\hat{y})=\frac{\frac{2 \hat{y}^{2}}{y_{\max }}-\frac{4}{3} \frac{\hat{y}^{3}}{y_{\max }^{2}}-\frac{y_{\max }}{6}}{\frac{4 \hat{y}}{y_{\max }}\left(1-\frac{\hat{y}}{2 y_{\max }}\right)-1}
$$

and

$$
\bar{E}(\hat{y})=\frac{\frac{2 y_{\max }}{3}-\frac{2 \hat{y}^{2}}{y_{\max }}+\frac{4}{3} \frac{\hat{y}^{3}}{y_{\max }^{2}}}{2-\frac{4 \hat{y}}{y_{\max }}+\frac{2 \hat{y}^{2}}{y_{\max }^{2}}}
$$

Monopolist profits are

$$
\Pi(\hat{y})=\hat{y}(E-\underline{E}(\hat{y})) .
$$

It is straightforward to show that $\Pi($.$) reaches a local maximum at \frac{3 y_{\max }}{8}$ if $\hat{y} \leq \frac{y_{\max }}{2}$ and is decreasing in $\hat{y}$ for all $\hat{y}>\frac{y_{\max }}{2}$. Hence, cutoff $\hat{y}^{*}=\frac{3 y_{\max }}{8}$ yields the maximal profit, and $\Pi\left(\hat{y}^{*}\right)=\frac{3}{32} y_{\max }^{2}$. Welfare at this cutoff is given by

$U^{S}\left(\hat{y}^{*}\right)=F\left(\hat{y}^{*}\right)\left(\underline{E}\left(\hat{y}^{*}\right)\right)^{2}+\left(1-F\left(\hat{y}^{*}\right)\right)\left(\bar{E}\left(\hat{y}^{*}\right)\right)^{2}-\Pi\left(\hat{y}^{*}\right)=\frac{y_{\max }^{2}}{32}\left(\frac{3059}{529}\right) \approx 0.1807 y_{\max }^{2}$

\subsubsection{Uniform distribution}

We have that

$$
\underline{E}(\hat{y})=\frac{\hat{y}}{2}
$$

and

$$
\bar{E}(\hat{y})=\frac{y_{\max }+\hat{y}}{2}
$$

and thus

$$
\Pi(\hat{y})=\frac{y_{\max } \hat{y}}{2}-\frac{\hat{y}^{2}}{2}
$$

which is maximized at

$$
\hat{y}^{*}=\frac{y_{\max }}{2} .
$$

Total welfare at $\hat{y}^{*}$ is

$$
U^{S}\left(\hat{y}^{*}\right)=\frac{3 y_{\max }^{2}}{16} .
$$




\subsubsection{Reverse triangle distribution}

If the density is

$$
\begin{aligned}
& f(y)=\frac{2}{y_{\max }}-\frac{4}{\left(y_{\max }\right)^{2}} y \quad \text { if } y \in\left[0, \frac{y_{\max }}{2}\right] \\
& f(y)=-\frac{2}{y_{\max }}+\frac{4}{\left(y_{\max }\right)^{2}} y \quad \text { if } y \in\left[\frac{y_{\max }}{2}, y_{\max }\right]
\end{aligned}
$$

then I can calculate that if the cutoff $\hat{y}$ is in $\left[0, \frac{y_{\max }}{2}\right]$ the conditional expectations are

$$
\underline{E}(\hat{y})=\frac{\frac{\hat{y}}{2}-\frac{2}{3} \frac{\hat{y}^{2}}{y_{\max }}}{1-\frac{\hat{y}}{y_{\max }}}
$$

and

$$
\bar{E}(\hat{y})=\frac{\frac{y_{\max }}{2}-\frac{2 \hat{y}}{y_{\max }}\left(\frac{\hat{y}}{2}-\frac{2}{3} \frac{\hat{y}^{2}}{y_{\max }}\right)}{1-\frac{2 \hat{y}}{y_{\max }}\left(1-\frac{\hat{y}}{y_{\max }}\right)}
$$

whereas if $\hat{y} \in\left[\frac{y_{\max }}{2}, y_{\max }\right]$ the expressions become

$$
\underline{E}(\hat{y})=\frac{\frac{y_{\max }}{6}-\frac{2 \hat{y}^{2}}{y_{\max }}\left(\frac{1}{2}-\frac{2}{3} \frac{\hat{y}}{y_{\max }}\right)}{1-\frac{2 \hat{y}}{y_{\max }}\left(1-\frac{\hat{y}}{y_{\max }}\right)}
$$

and

$$
\bar{E}(\hat{y})=\frac{\frac{y_{\max }}{3}+\frac{2 \hat{y}^{2}}{y_{\max }}\left(\frac{1}{2}-\frac{2}{3} \frac{\hat{y}}{y_{\max }}\right)}{\frac{2 \hat{y}}{y_{\max }}\left(1-\frac{\hat{y}}{y_{\max }}\right)} .
$$

Monopolist profits are

$$
\Pi(\hat{y})=\hat{y}(E-\underline{E}(\hat{y}))
$$

It is straightforward to show that $\Pi($.$) reaches a local maximum at 0.64 y_{\max }$ (numerically calculated) if $\hat{y}>\frac{y_{\max }}{2}$ and is decreasing in $\hat{y}$ for all $\hat{y} \leq$ $\frac{y_{\max }}{2}$. Hence, cutoff $\hat{y}^{*}=0.64 y_{\max }$ yields the maximal profit, and $\Pi\left(\hat{y}^{*}\right) \approx$ $0.1935 y_{\max }^{2}$. Welfare at this cutoff is given by

$$
U^{S}\left(\hat{y}^{*}\right)=F\left(\hat{y}^{*}\right)\left(\underline{E}\left(\hat{y}^{*}\right)\right)^{2}+\left(1-F\left(\hat{y}^{*}\right)\right)\left(\bar{E}\left(\hat{y}^{*}\right)\right)^{2}-\Pi\left(\hat{y}^{*}\right) \approx 0.163 y_{\max }^{2}
$$




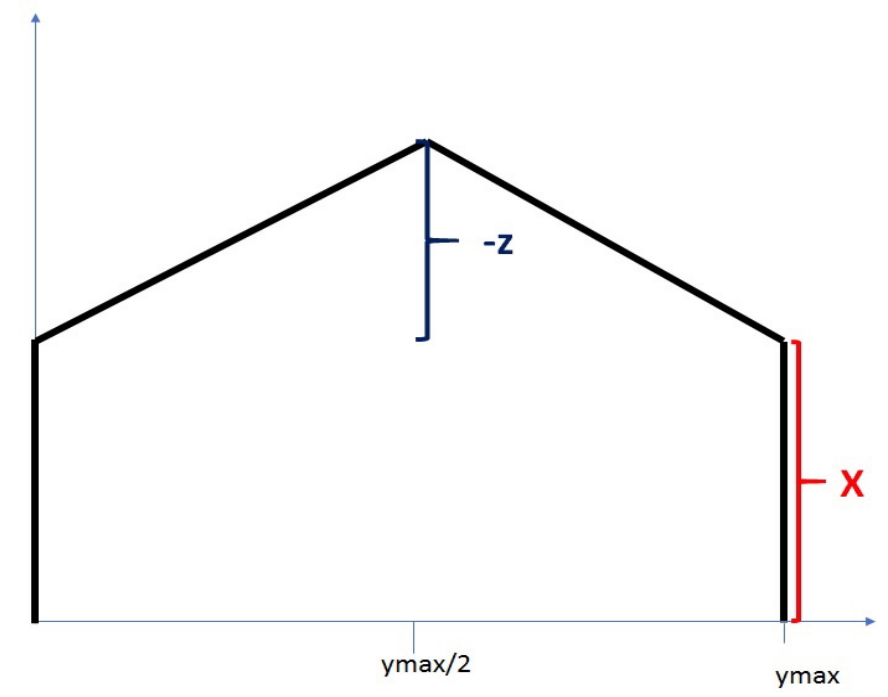

Figure 1: House distribution

\subsection{House distribution, uniform distribution, trough distribution}

Suppose income is distributed according to an income distribution with pdf $f($.$) such that$

$$
\begin{aligned}
& f(y)=x-\frac{2 z}{y_{\max }} y \quad \text { if } y \in\left[0, \frac{y_{\max }}{2}\right] \\
& f(y)=x-2 z+\frac{2 z}{y_{\max }} y \quad \text { if } y \in\left[\frac{y_{\max }}{2}, y_{\max }\right]
\end{aligned}
$$

Note that we must have

$$
x=\frac{1}{y_{\max }}+\frac{z}{2}
$$

in order for $F\left(y_{\max }\right)=1$ and

$$
x \in\left[0, \frac{2}{y_{\max }}\right], \quad z \in\left[-\frac{2}{y_{\max }}, \frac{2}{y_{\max }}\right]
$$

If $z=0$ then $x=\frac{1}{y_{\max }}$ and the distribution is uniform, if $z=-\frac{2}{y_{\max }}$ then $x$ is 0 and the pdf has the shape of an isosceles triangle. If $z=\frac{2}{y_{\max }}$ then $x=\frac{2}{y_{\max }}$ and the pdf has the shape of an inverse triangle. If $z \in$ 


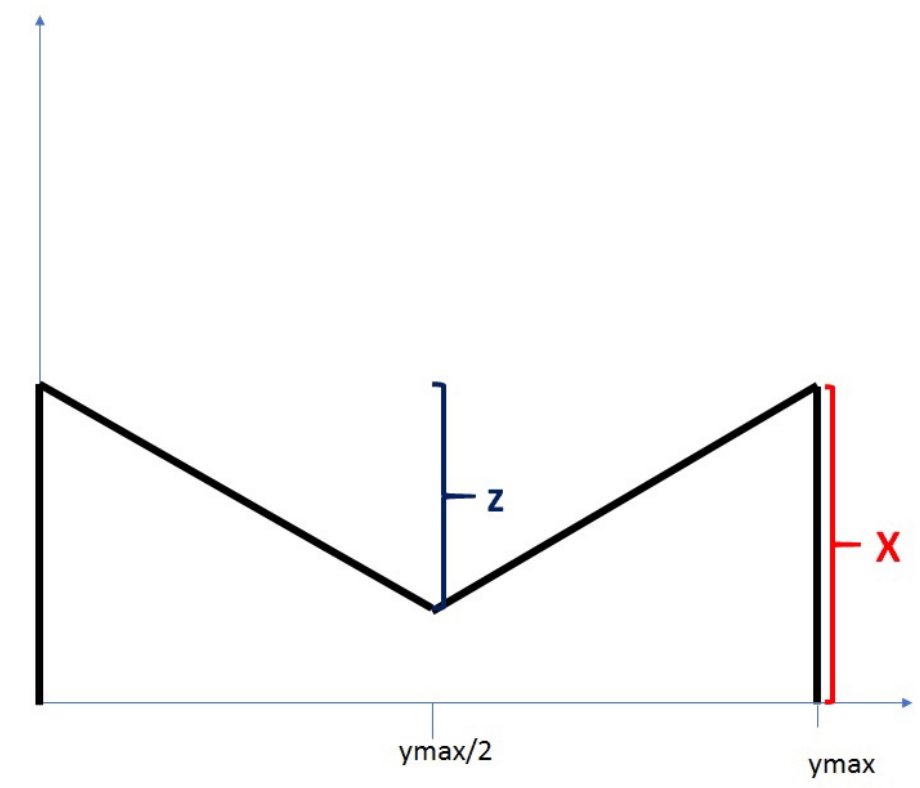

Figure 2: Trough distribution

$\left(-\frac{2}{y_{\max }}, 0\right)$ then the distribution has the shape of a house, if $z \in\left(0, \frac{2}{y_{\max }}\right)$ the distribution has the shape of a (triangular) trough (see Figures 1 and 2). The larger $z$, the higher is inequality (in terms of $\bar{E}-\underline{E}$ for any given cutoff) and an increase in $z$ amounts to a mean-preserving spread of the income distribution. Note that average income is constant $\forall z \in\left[-\frac{2}{y_{\max }}, \frac{2}{y_{\max }}\right]$, $E(y)=\frac{y_{\max }}{2}$.

Using (10), the pdf can be rewritten as

$$
\begin{array}{ll}
f(y)=\frac{1}{y_{\max }}+\frac{z}{2}-\frac{2 z}{y_{\max }} y & \text { if } y \in\left[0, \frac{y_{\max }}{2}\right] \\
f(y)=\frac{1}{y_{\max }}-\frac{3 z}{2}+\frac{2 z}{y_{\max }} y & \text { if } y \in\left[\frac{y_{\max }}{2}, y_{\max }\right]
\end{array}
$$

If the cutoff $\hat{y}$ is in the interval $\left[0, \frac{y_{\max }}{2}\right]$, we have that

$$
\underline{E}(\hat{y})=\frac{\int_{0}^{\hat{y}}\left(\frac{1}{y_{\max }}+\frac{z}{2}-\frac{2 z}{y_{\max }} y\right) y d y}{F(\hat{y})}=\frac{\int_{0}^{\hat{y}}\left(\frac{1}{y_{\max }}+\frac{z}{2}-\frac{2 z}{y_{\max }} y\right) y d y}{\int_{0}^{\hat{y}}\left(\frac{1}{y_{\max }}+\frac{z}{2}-\frac{2 z}{y_{\max }} y\right) d y}
$$




$$
=\frac{\frac{\hat{y}^{2}}{2 y_{\max }}+\frac{\hat{y}^{2} z}{4}-\frac{2 z \hat{y}^{3}}{3 y_{\max }}}{\frac{\hat{y}(1-z \hat{y})}{y_{\max }}+\frac{\hat{y} z}{2}}=\frac{6 \hat{y}+3 \hat{y} z y_{\max }-8 z \hat{y}^{2}}{12-12 z \hat{y}+6 y_{\max } z}
$$

If the cutoff is above $\frac{y_{\max }}{2}$ we need to calculate $\underline{E}(\hat{y})$ differently: The easiest way is to calculate $\bar{E}(\hat{y})$ first

$$
\begin{gathered}
\bar{E}(\hat{y})=\frac{\int_{\hat{y}}^{y_{\max }}\left(\frac{1}{y_{\max }}-\frac{3 z}{2}+\frac{2 z}{y_{\max }} y\right) y d y}{1-F(\hat{y})}=\frac{\int_{\hat{y}}^{y_{\max }}\left(\frac{1}{y_{\max }}-\frac{3 z}{2}+\frac{2 z}{y_{\max }} y\right) y d y}{\int_{\hat{y}}\left(\frac{1}{y_{\max }}-\frac{3 z}{2}+\frac{2 z}{y_{\max }} y\right) d y} \\
=\frac{\frac{y_{\max }}{2}-\frac{1}{12} z y_{\max }^{2}-\frac{\hat{y}^{2}}{2 y_{\max }}+\frac{3}{4} z \hat{y}^{2}-\frac{2}{3} \frac{z}{y_{\max }} \hat{y}^{3}}{1-\frac{1}{2} z y_{\max }-\frac{\hat{y}}{y_{\max }}+\frac{3 z \hat{y}}{2}-\frac{z \hat{y}^{2}}{y_{\max }}}
\end{gathered}
$$

and then calculate $\underline{E}(\hat{y})$ via the formula

$$
E=F(\hat{y}) \underline{E}(\hat{y})+(1-F(\hat{y})) \bar{E}(\hat{y})
$$

(noting that $E=\frac{y_{\max }}{2}$ ), which gives

$$
\begin{aligned}
E(\hat{y}) & =\frac{\frac{1}{12} z y_{\max }^{2}+\frac{\hat{y}^{2}}{2 y_{\max }}-\frac{3}{4} z \hat{y}^{2}+\frac{2}{3} \frac{z}{y_{\max }} \hat{y}^{3}}{\frac{1}{2} z y_{\max }+\frac{\hat{y}}{y_{\max }}-\frac{3 z \hat{y}}{2}+\frac{z \hat{y}^{2}}{y_{\max }}} \\
& =\frac{z y_{\max }^{2}+\frac{6 \hat{y}^{2}}{y_{\max }}-9 z \hat{y}^{2}+\frac{8 z \hat{y}^{3}}{y_{\max }}}{6 z y_{\max }+\frac{12 \hat{y}}{y_{\max }}-18 z \hat{y}+\frac{12 \hat{y}^{2} z}{y_{\max }}} .
\end{aligned}
$$

Using these expressions, I can show the following:

Proposition 21 If $z \in\left[-\frac{2}{y_{\max }}, 0\right]$, the monopolist's optimal cutoff is in the interval $\left[0, \frac{y_{\max }}{2}\right]$, if $z=0$ the monopolist's optimal cutoff is $\hat{y}^{*}=$ $\frac{y_{\max }}{2}$ and if $z \in\left[0, \frac{2}{y_{\max }}\right]$, the monopolist's optimal cutoff is in the interval $\left[\frac{y_{\max }}{2}, y_{\max }\right]$.

Proof. The monopolist's profit at cutoff $\hat{y}$ is given by

$$
\Pi(\hat{y})=\hat{y}(E-\underline{E})
$$


Using the expressions for $\underline{E}$ from above, I find that

$$
\Pi(\hat{y})=\hat{y}\left(\frac{y_{\max }}{2}-\frac{6 \hat{y}+3 \hat{y} z y_{\max }-8 z \hat{y}^{2}}{12-12 z \hat{y}+6 y_{\max } z}\right)
$$

if $\hat{y} \in\left[0, \frac{y_{\max }}{2}\right]$ and

$$
\Pi(\hat{y})=\hat{y}\left(\frac{y_{\max }}{2}-\frac{z y_{\max }^{2}+\frac{6 \hat{y}^{2}}{y_{\max }}-9 z \hat{y}^{2}+\frac{8 z \hat{y}^{3}}{y_{\max }}}{6 z y_{\max }+\frac{12 \hat{y}}{y_{\max }}-18 z \hat{y}+\frac{12 \hat{y}^{2} z}{y_{\max }}}\right)
$$

if $\hat{y} \in\left[\frac{y_{\max }}{2}, y_{\max }\right]$. It can be calculated (numerically) that (11) has a local and global maximum in $\left[0, \frac{y_{\max }}{2}\right]$ when $z<0$, while (12) has a local and global maximum in $\left[\frac{y_{\max }}{2}, y_{\max }\right]$ when $z>0$.

Proposition 22 The monopolist's profit-maximizing cutoff $\hat{y}^{*}$ is increasing in $z$ for all $z \in\left[-\frac{2}{y_{\max }}, \frac{2}{y_{\max }}\right]$.

Proof. Note that

$$
\frac{d \hat{y}^{*}}{d z}=\frac{-\frac{\partial \underline{E}\left(\hat{y}^{*}, z\right)}{\partial z}-\hat{y}^{*} \frac{\partial^{2} \underline{E}\left(\hat{y}^{*}, z\right)}{\partial \hat{y} \partial z}}{\hat{y}^{*} \frac{\partial^{2} \underline{E}(\hat{y}, z)}{(\partial \hat{y})^{2}}+2 \frac{\partial \underline{\underline{E}}\left(\hat{y}^{*}, z\right)}{\partial \hat{y}}}
$$

and hence according to Lemma 1, sufficient conditions for

$$
\frac{d \hat{y}^{*}}{d z}>0
$$

are

$$
\frac{\partial^{2} \underline{E}(\hat{y}, z)}{\partial \hat{y} \partial z} \leq 0 \quad \text { and } \quad \frac{\partial^{2} \underline{E}(\hat{y}, z)}{(\partial \hat{y})^{2}} \geq 0 .
$$

Show $\frac{\partial^{2} E(\hat{y}, z)}{(\partial \hat{y})^{2}} \geq 0$ if $z<0$ :

If $z<0$ we know that the monopolist's optimal cutoff is in the interval $\left[0, \frac{y_{\max }}{2}\right)$. Setting $y_{\max }=1$ again for simplicity of notation we have that

$$
\begin{gathered}
\underline{E}(\hat{y}, z)=\frac{6 \hat{y}+3 \hat{y} z-8 z \hat{y}^{2}}{12-12 z \hat{y}+6 z} \\
\frac{\partial \underline{E}(\hat{y}, z)}{\partial \hat{y}}=\frac{(6+3 z-16 z \hat{y})(12-12 z \hat{y}+6 z)+\left(6 \hat{y}+3 \hat{y} z-8 z \hat{y}^{2}\right) 12 z}{(12-12 z \hat{y}+6 z)^{2}} \\
=6 \frac{12+12 z-32 z \hat{y}-16 z^{2} \hat{y}+16 z^{2} \hat{y}^{2}+3 z^{2}}{(12-12 z \hat{y}+6 z)^{2}}
\end{gathered}
$$


Therefore

$\frac{\partial^{2} \underline{E}(\hat{y}, z)}{(\partial \hat{y})^{2}}=6 \cdot \frac{\left(-32 z-16 z^{2}+32 z^{2} \hat{y}\right)(12-12 z \hat{y}+6 z)+24 z\left(12+12 z-32 z \hat{y}-16 z^{2} \hat{y}+16 z^{2} \hat{y}^{2}\right.}{(12-12 z \hat{y}+6 z)^{3}}$

It is immediate to see that

$$
12-12 z \hat{y}+6 z>0
$$

for all $z<0$, i.e. $z \in[-2,0]$, therefore it suffices to examine the numerator of this expression. The numerator can be rewritten as

$$
36\left(-16 z-16 z^{2}-4 z^{3}\right)
$$

which is always positive if $z<0$. I have therefore demonstrated that $\frac{\partial^{2} \underline{E}(\hat{y}, z)}{(\partial \hat{y})^{2}}>0$ if $z<0$.

Show $\frac{\partial^{2} E(\hat{y}, z)}{\partial \hat{y} \partial z} \leq 0$ if $z<0$ :

Given that

$$
\frac{\partial \underline{E}(\hat{y}, z)}{\partial \hat{y}}=6 \frac{12+12 z-32 z \hat{y}-16 z^{2} \hat{y}+16 z^{2} \hat{y}^{2}+3 z^{2}}{(12-12 z \hat{y}+6 z)^{2}}
$$

I can calculate

$\frac{\partial^{2} \underline{E}(\hat{y}, z)}{\partial \hat{y} \partial z}=6 \frac{\left[\begin{array}{c}\left(12-32 \hat{y}-32 z \hat{y}+32 z \hat{y}^{2}+6 z\right)(12-12 z \hat{y}+6 z) \\ -2(-12 \hat{y}+6)\left(12+12 z-32 z \hat{y}-16 z^{2} \hat{y}+16 z^{2} \hat{y}^{2}+3 z^{2}\right)\end{array}\right]}{(12-12 z \hat{y}+6 z)^{3}}$

Again it suffices to examine the numerator, which can be rewritten as

$$
\begin{aligned}
& 36\left[\left(12-32 \hat{y}-32 z \hat{y}+32 z \hat{y}^{2}+6 z\right)(2-2 z \hat{y}+z)\right. \\
& \left.-(-4 \hat{y}+2)\left(12+12 z-32 z \hat{y}-16 z^{2} \hat{y}+16 z^{2} \hat{y}^{2}+3 z^{2}\right)\right]
\end{aligned}
$$

and simplified to

$$
36 \hat{y}(-16-8 z)
$$

which is always negative if $z \in[-2,0]$. I have therefore demonstrated that $\frac{\partial^{2} \underline{E}(\hat{y}, z)}{\partial \hat{y} \partial z}<0$ if $z<0$.

As both sufficient conditions hold, we have that $\frac{d \hat{y}^{*}}{d z}>0$ if $z<0$. 
Show $\frac{\partial^{2} \underline{E}(\hat{y}, z)}{(\partial \hat{y})^{2}} \geq 0$ if $z>0$ :

If $z>0$ we know the monopolist's optimal cutoff lies above $\frac{y_{\max }}{2}$ and therefore (again setting $y_{\max }=1$ )

$$
\underline{E}(\hat{y}, z)=\frac{z+6 \hat{y}^{2}-9 z \hat{y}^{2}+8 z \hat{y}^{3}}{6 z+12 \hat{y}-18 z \hat{y}+12 \hat{y}^{2} z}
$$

Therefore we have

$$
\begin{aligned}
\frac{\partial \underline{E}(\hat{y}, z)}{\partial \hat{y}}= & \frac{6}{\left(6 z+12 \hat{y}-18 z \hat{y}+12 \hat{y}^{2} z\right)^{2}}\left[-2 z+3 z^{2}+12 \hat{y}^{2}+12 z y-22 z^{2} \hat{y}+51 z^{2} \hat{y}^{2}\right. \\
& \left.-36 z \hat{y}^{2}-48 z^{2} \hat{y}^{3}+16 z^{2} \hat{y}^{4}+32 z \hat{y}^{3}\right]
\end{aligned}
$$

and

$$
\begin{aligned}
\frac{\partial^{2} \underline{E}(\hat{y}, z)}{(\partial \hat{y})^{2}}= & \frac{36}{\left(6 z+12 \hat{y}-18 z \hat{y}+12 \hat{y}^{2} z\right)^{3}} \times \\
& {\left[\left(24 \hat{y}+12 z-22 z^{2}+102 z^{2} \hat{y}-72 z \hat{y}-144 z^{2} \hat{y}^{2}+64 z^{2} \hat{y}^{3}+96 z \hat{y}^{2}\right)(z+2 \hat{y}-3 z \hat{y}\right.} \\
& +(-4+6 z-8 z \hat{y})\left(-2 z+3 z^{2}+12 \hat{y}^{2}+12 z y-22 z^{2} \hat{y}\right. \\
& \left.\left.+51 z^{2} \hat{y}^{2}-36 z \hat{y}^{2}-48 z^{2} \hat{y}^{3}+16 z^{2} \hat{y}^{4}+32 z \hat{y}^{3}\right)\right]
\end{aligned}
$$

which can be shown to be positive $\forall y \in[0.5,1]$ and $\forall z>0$.

Show $\frac{\partial^{2} E(\hat{y}, z)}{\partial \hat{y} \partial z} \leq 0$ if $z>0$ :

$$
\frac{\partial \underline{E}(\hat{y}, z)}{\partial z}=\frac{6\left(2 \hat{y}+4 \hat{y}^{4}-6 \hat{y}^{2}\right)}{\left(6 z+12 \hat{y}-18 z \hat{y}+12 \hat{y}^{2} z\right)^{2}}
$$

and hence

$$
\begin{aligned}
\frac{\partial^{2} \underline{E}(\hat{y}, z)}{\partial z \partial y}= & \frac{36}{\left(6 z+12 \hat{y}-18 z \hat{y}+12 \hat{y}^{2} z\right)^{3}}\left[\left(2+16 \hat{y}^{3}-12 \hat{y}\right)\left(z+2 \hat{y}-3 z \hat{y}+2 z \hat{y}^{2}\right)\right. \\
& \left.-2\left(2 \hat{y}+4 \hat{y}^{4}-6 \hat{y}^{2}\right)(2-3 z+4 z \hat{y})\right]
\end{aligned}
$$

Unfortunately, this is not always negative. In fact for high $\hat{y}$ it can be seen from simulations that it is positive for all $z$. The intuition for this is that the shape of the distribution is that of a trough in this case, and as $z$ increases the trough becomes deeper. This means that there is a lot of mass higher up in the income distribution, and as the cutoff moves towards there, average income in the poor group increases due to this. This means 
that one of the sufficient conditions doesn't hold in the case of $z>0$, so we need to calculate the whole expression for $\frac{d \hat{y}}{d z}$ to prove that it is positive. Plugging all the derivatives into this expression yields indeed that $\frac{d \hat{y}^{*}}{d z}>0$ for all $z$ (numerically calculated - note that the maximum $\hat{y}^{*}$ is at 0.6427051 , when $z=-2)$.

We already know from Proposition 1 that an increase in inequality (resp. $z$ ) increases the monopolist's maximized profits. But what happens to total welfare, welfare of the richest and average welfare in the poor group?

Proposition 23 Welfare from sorting at the monopolist's optimal cutoff is increasing in $z$ if $z \in\left[-\frac{2}{y_{\max }}, 0\right]$.

Proof. The derivative of total welfare with respect to $z$ at the monopolist's optimal cutoff $\hat{y}^{*}$ amounts to

$$
\begin{gathered}
\frac{d T W\left(\hat{y}^{*}, z\right)}{d z}=\left(f\left(\underline{E}^{2}-\bar{E}^{2}\right)+F 2 \underline{E} \frac{\partial \underline{E}}{\partial \hat{y}^{*}}+(1-F) 2 \bar{E} \frac{\partial \bar{E}}{\partial \hat{y}^{*}}\right) \frac{d \hat{y}^{*}}{d z} \\
+\frac{\partial F}{\partial z}\left(\underline{E}^{2}-\bar{E}^{2}\right)+F 2 \underline{E} \frac{\partial \underline{E}}{\partial z}+(1-F) 2 \bar{E} \frac{\partial \bar{E}}{\partial z}-\frac{d \Pi\left(\hat{y}^{*}, z\right)}{d z}
\end{gathered}
$$

where $\Pi\left(\hat{y}^{*}, z\right)$ is the monopolist's maximized profit and we know that

$$
\frac{d \Pi\left(\hat{y}^{*}, z\right)}{d z}=-\hat{y}^{*} \frac{\partial \underline{E}}{\partial z}>0
$$

Hence, the above expression can be simplified to

$$
\begin{gathered}
\frac{d T W\left(\hat{y}^{*}, z\right)}{d z}=f(\bar{E}-\underline{E})\left(\bar{E}+\underline{E}-2 \hat{y}^{*}\right) \frac{d \hat{y}^{*}}{d z}+ \\
+\frac{\partial F}{\partial z}\left(\underline{E}^{2}-\bar{E}^{2}\right)+\left(F 2 \underline{E}+\hat{y}^{*}\right) \frac{\partial \underline{E}}{\partial z}+(1-F) 2 \bar{E} \frac{\partial \bar{E}}{\partial z}
\end{gathered}
$$

Note that if $z<0$ we know that $\hat{y}^{*}<\frac{y_{\max }}{2}$.

(Set $y_{\max }=1$ again) We have

$$
\bar{E}=\frac{6-6 \hat{y}^{2}-3 z \hat{y}^{2}+8 z \hat{y}^{3}}{12-12 \hat{y}-6 z \hat{y}+12 z \hat{y}^{2}}
$$

and

$$
\frac{\partial \bar{E}}{\partial z}=\frac{6}{\left(12-12 \hat{y}-6 z \hat{y}+12 z \hat{y}^{2}\right)^{2}} \hat{y}\left(6-18 \hat{y}-4 \hat{y}^{3}+16 \hat{y}^{2}\right)
$$


Furthermore

$$
\frac{\partial \underline{E}}{\partial z}=\frac{6}{(12-6 z+12 z \hat{y})^{2}} \hat{y}\left(-4 \hat{y}^{2}\right)
$$

and note that

$$
F(\hat{y}, z)=\hat{y}+\frac{z \hat{y}}{2}-z \hat{y}^{2}
$$

and hence

$$
\frac{\partial F}{\partial z}=\frac{\hat{y}}{2}-\hat{y}^{2}>0 \quad \forall \hat{y} \in[0,0.5]
$$

Note that $\frac{\partial E}{\partial z}<0$ but $\frac{\partial F}{\partial z}>0$ because $\hat{y}^{*}<\frac{y_{\max }}{2}$. As I have shown above that $\frac{d \hat{y}^{*}}{d z}>0 \forall z$, sufficient conditions for $\frac{d T W\left(\hat{y}^{*}, z\right)}{d z}>0$ are that

$$
\bar{E}+\underline{E}-2 \hat{y}^{*}>0
$$

and

$$
\frac{\partial F}{\partial z}\left(\underline{E}^{2}-\bar{E}^{2}\right)+\left(F 2 \underline{E}+\hat{y}^{*}\right) \frac{\partial \underline{E}}{\partial z}+(1-F) 2 \bar{E} \frac{\partial \bar{E}}{\partial z}>0
$$

It is easy to check that condition (13) always holds in this case. After some algebra, it can be seen from numerical calculations that also (14) holds. Hence, $\frac{d T W\left(\hat{y}^{*}, z\right)}{d z}>0$ if $z<0$.

Proposition 24 Welfare from sorting at the monopolist's optimal cutoff is decreasing in $z$ if $z \in\left[0, \frac{2}{y_{\max }}\right]$.

Proof. Note that if $z>0$ we know that $\hat{y}>\frac{y_{\max }}{2}$. As above we have

$$
\begin{gathered}
\frac{d T W\left(\hat{y}^{*}, z\right)}{d z}=f(\bar{E}-\underline{E})\left(\bar{E}+\underline{E}-2 \hat{y}^{*}\right) \frac{d \hat{y}^{*}}{d z}+ \\
+\frac{\partial F}{\partial z}\left(\underline{E}^{2}-\bar{E}^{2}\right)+\left(F 2 \underline{E}+\hat{y}^{*}\right) \frac{\partial \underline{E}}{\partial z}+(1-F) 2 \bar{E} \frac{\partial \bar{E}}{\partial z}
\end{gathered}
$$

Sufficient conditions for $\frac{d T W\left(\hat{y}^{*}, z\right)}{d z}<0$ are that

$$
\bar{E}+\underline{E}-2 \hat{y}^{*}<0
$$

and

$$
\frac{\partial F}{\partial z}\left(\underline{E}^{2}-\bar{E}^{2}\right)+\left(F 2 \underline{E}+\hat{y}^{*}\right) \frac{\partial \underline{E}}{\partial z}+(1-F) 2 \bar{E} \frac{\partial \bar{E}}{\partial z}<0 .
$$


Note that in this case we have (again setting $y_{\max }=1$ ) that

$$
\bar{E}=\frac{6-z-6 \hat{y}^{2}+9 z \hat{y}^{2}-8 z \hat{y}^{3}}{12-6 z-12 \hat{y}+18 z \hat{y}-12 z \hat{y}^{2}}
$$

and

$$
\underline{E}=\frac{z+6 \hat{y}^{2}-9 z \hat{y}^{2}+8 z \hat{y}^{3}}{6 z+12 \hat{y}-18 z \hat{y}+12 \hat{y}^{2} z}
$$

Hence

$$
\frac{\partial \underline{E}}{\partial z}=\frac{6}{\left(6 z+12 \hat{y}-18 z \hat{y}+12 \hat{y}^{2} z\right)^{2}}\left(2 \hat{y}+4 \hat{y}^{4}-6 \hat{y}^{2}\right)
$$

and

$$
\frac{\partial \bar{E}}{\partial z}=\frac{6}{\left(12-6 z-12 \hat{y}+18 z \hat{y}-12 z \hat{y}^{2}\right)^{2}}\left(4-16 \hat{y}-16 \hat{y}^{3}+24 \hat{y}^{2}+16 \hat{y}^{4}\right) .
$$

Furthermore, note that

$$
F(\hat{y}, z)=\frac{1}{2} z+\hat{y}-\frac{3 z \hat{y}}{2}+z \hat{y}^{2}
$$

and hence

$$
\frac{\partial F}{\partial z}=\frac{1}{2}-\frac{3 \hat{y}}{2}+\hat{y}^{2}
$$

Plugging in these expressions, it can easily be shown that (15) is always negative. However, concerning (16), there is a small range of $\hat{y}>0.5$ for which this expression is positive. Indeed it can be shown (in numerical simulations) that the whole expression $\frac{d T W\left(\hat{y}^{*}, z\right)}{d z}$ is positive for all $z$ for small $\hat{y}^{*}>0.5$. However, note that the monopolist's optimal cutoff increases with $z$ as well, and this increase moves $\hat{y}^{*}$ out of the area for which total welfare increases with $z$ also for very small $z$. In fact, for all $z>0$ it can be shown (again numerically) that $\hat{y}^{*}$ is greater than the small range of $\hat{y}$ for which $\frac{d T W\left(\hat{y}^{*}, z\right)}{d z}$ would be positive. Hence, total welfare from sorting at the monopolist's optimal cutoff decreases with $z$ if $z>0$.

Proposition 25 Welfare of the richest from sorting at the monopolist's optimum is increasing in z for low rates of inequality and decreasing in $z$ for high rates of inequality.

Proof.

$$
W_{y_{\max }}\left(\hat{y}^{*}, z\right)=\left(y_{\max }-\hat{y}^{*}\right) \bar{E}\left(\hat{y}^{*}, z\right)+\hat{y}^{*} \underline{E}\left(\hat{y}^{*}, z\right)
$$


The monopolist's optimal cutoff satisfies the FOC and hence

$$
E-\underline{E}\left(\hat{y}^{*}, z\right)=\hat{y}^{*} \frac{\partial \underline{E}\left(\hat{y}^{*}, z\right)}{\partial \hat{y}}
$$

The derivative of $W_{y_{\max }}\left(\hat{y}^{*}\right)$ with respect to $z$ is:

$$
\frac{d W_{y_{\max }}\left(\hat{y}^{*}, z\right)}{d z}=\left(y_{\max }-\hat{y}^{*}\right)\left(\frac{\partial \bar{E}}{\partial z}\right)+\hat{y}^{*} \frac{\partial \underline{E}}{\partial z}+\left[\left(y_{\max }-\hat{y}^{*}\right) \frac{\partial \bar{E}}{\partial \hat{y}}-(\bar{E}-\underline{E})+\hat{y}^{*} \frac{\partial \underline{E}}{\partial \hat{y}}\right] \frac{d \hat{y}^{*}}{d z}
$$

Using (17) this becomes

$$
\frac{d W_{y_{\max }}\left(\hat{y}^{*}, z\right)}{d z}=\left(y_{\max }-\hat{y}^{*}\right)\left(\frac{\partial \bar{E}}{\partial z}\right)+\hat{y}^{*} \frac{\partial \underline{E}}{\partial z}+\left[\left(y_{\max }-\hat{y}^{*}\right) \frac{\partial \bar{E}}{\partial \hat{y}}-(\bar{E}-E)\right] \frac{d \hat{y}^{*}}{d z}
$$

Hence, sufficient conditions for $W_{y_{\max }}\left(\hat{y}^{*}\right)$ to be decreasing in $z$ are that

$$
\left(y_{\max }-\hat{y}^{*}\right)\left(\frac{\partial \bar{E}}{\partial z}\right)+\hat{y}^{*} \frac{\partial \underline{E}}{\partial z}<0
$$

and

$$
\left[\left(y_{\max }-\hat{y}^{*}\right) \frac{\partial \bar{E}}{\partial \hat{y}}-(\bar{E}-E)\right]<0
$$

(because we already know that $\frac{d \hat{y}^{*}}{d z}>0$ ).

For $z>0$ it can be shown (numerically) that both terms are negative for $\hat{y}^{*} \in[0.5,1]$ and all $z \in[0,0.5]$. Hence, $\frac{d W_{y \max }\left(\hat{y}^{*}, z\right)}{d z}$ is negative for all $\hat{y}$ in $[0.5,1]$ for all $z$ and thus $W_{y_{\max }}\left(\hat{y}^{*}, z\right)$ is decreasing in $z$ : As inequality increases, welfare of the richest in society from sorting at the monopolist's optimal cutoff goes down.

For $z<0$ these sufficient conditions don't hold. In fact it can be shown (numerically) that except for very small $z<-1.9$, welfare of the richest in society from sorting at the monopolist's optimal cutoff increases due to an increase in inequality.

This last proposition helps in understanding the effect of an increase in inequality on the rich in the presence of sorting: as inequality increases, the monopolist increases the cutoff due to an increase in inequality, because the amount by which she can raise the sorting fee is higher than her loss of "customers" (= members of the rich group, who pay the fee). The increase in the cutoff benefits the rich group, but the increase in the 
sorting fee harms them. For low rates of inequality, the former effect is higher than the latter, hence welfare of the rich increases with inequality, but if inequality becomes too high (which, because it is in the form of a mean-preserving spread, means that there are more rich people as well as more poor) membership of their exclusive group becomes too expensive and the second effect dominates, leading to a negative relationship between inequality and welfare of the rich.

Proposition 26 Average welfare in the poor group from sorting at the monopolist's optimum decreases in inequality.

Proof. Average welfare in the poor group amounts to $\underline{E}^{2}$ (note that they don't have to pay $b$ ). We know that

$$
\frac{d \underline{E}\left(\hat{y}^{*}, z\right)}{d z}=\frac{\partial \underline{E}}{\partial \hat{y}^{*}} \frac{d \hat{y}^{*}}{d z}+\frac{\partial \underline{E}}{\partial z}
$$

Plugging in the expressions derived above, it is straightforward to show that (18) is negative for all $z$ and all $\hat{y}^{*}>0.5$. The intuition for this result is that, even though an increase in $\hat{y}$ actually benefits the poor group (because they get to interact with richer people on average), this is not enough to counteract the negative effect of an increasing mass of poor people with zero income in their group. The overall effect of an increase in inequality is thus negative.

\subsection{Lognormal distribution}

If we calibrate $\mu$ and $\sigma$ in the lognormal distribution to match the first and second moment of the US household distribution, we get $\mu \approx 10.85$ and $\sigma \approx 0.85$. I will thus often refer to these parameters in this section when comparing monopolist profits and welfare of sorting.

From numerical simulations, it can be concluded that the profit maximization problem of the monopolist always has a unique solution and the optimal cutoff is always increasing in $\sigma$. For instance, for $\mu=10.85$ and $\sigma=0.85$ the monopolist's profit as a function of the cutoff looks as in Figure 3. The vertical line marks the median of the underlying income distribution and therefore demonstrates that the optimal partition for the 


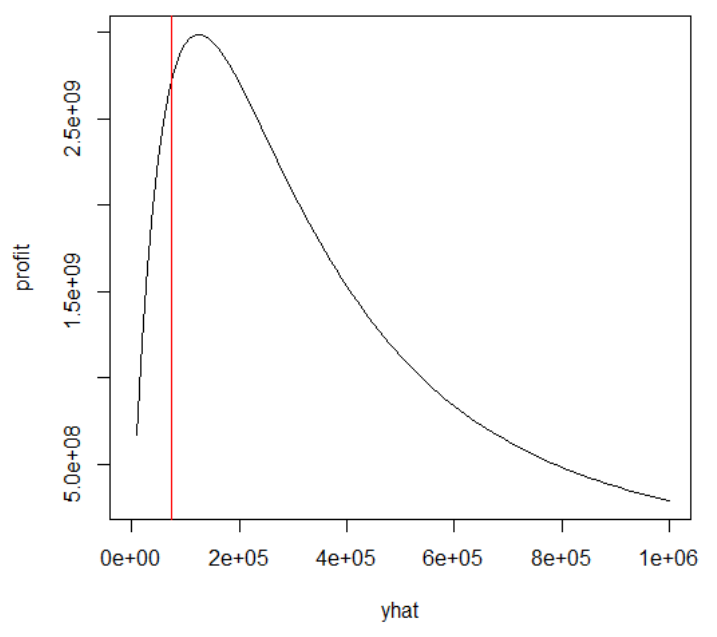

Figure 3: Monopolist profit as a function of the cutoff $\hat{y}$ (black) and median (red) if $\mu=10.85$ and $\sigma=0.85$

monopolist is such that the cutoff is above median (in fact it is even above average). If $\sigma$ declines, the optimal cutoff goes down and eventually will be below median income. If $\sigma$ increases, the opposite happens: the optimal cutoff increases.

If income is lognormally distributed with $\mu=10.85$ and $\sigma=0.85$, welfare as a function of the cutoff looks as in Figure 4. The optimal cutoff is above the median (and it can easily be seen that total welfare with sorting at this cutoff is higher than total welfare without sorting, which is the red line in the graph, $\left.E(y)^{2}\right)$. Note however, that for smaller $\sigma$ total welfare as a function of the cutoff looks differently. Figure 5 shows total welfare as a function of the cutoff for $\sigma=0.4$. Welfare is first declining in $\hat{y}$ and then increases again until it becomes flat and converges to the welfare of no sorting, $E(y)^{2}$. Therefore, no sorting is more efficient than sorting. Only once $\sigma$ increases above 0.65 does the shape change and a unique optimum $>0$ appears (see Figure 6 for the case where $\sigma=0.7$ ). As $\sigma$ increases further from then on, the welfare-maximizing cutoff increases. As the above analysis shows, monopolist profit-maximization and welfare maximization are not necessarily opposed goals in the case of the lognormal - indeed the optimal cutoffs in both cases are very close to each other and move in the 


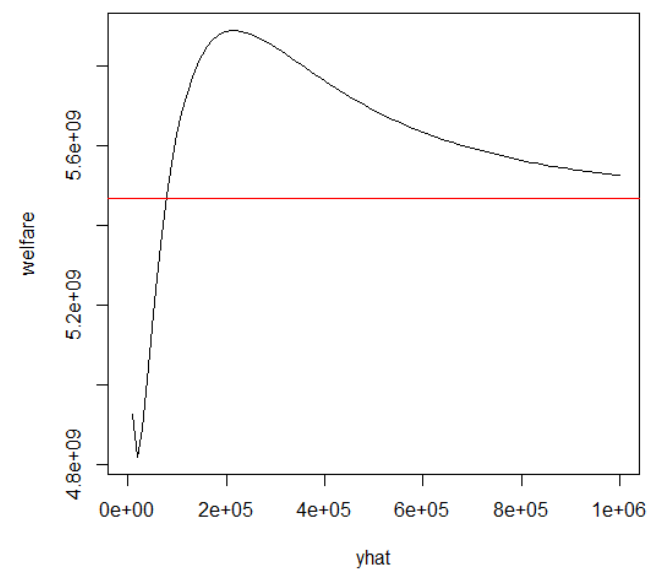

Figure 4: Welfare from sorting at cutoff $\hat{y}$ (black) and welfare without sorting (red) if $\mu=10.85$ and $\sigma=0.85$

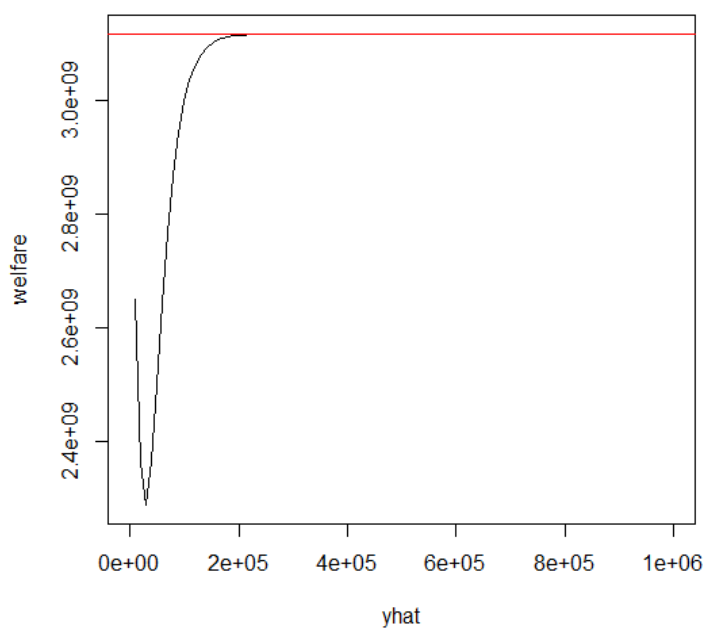

Figure 5: Welfare from sorting at cutoff $\hat{y}$ (black) and welfare without sorting (red) if $\mu=10.85$ and $\sigma=0.4$ 


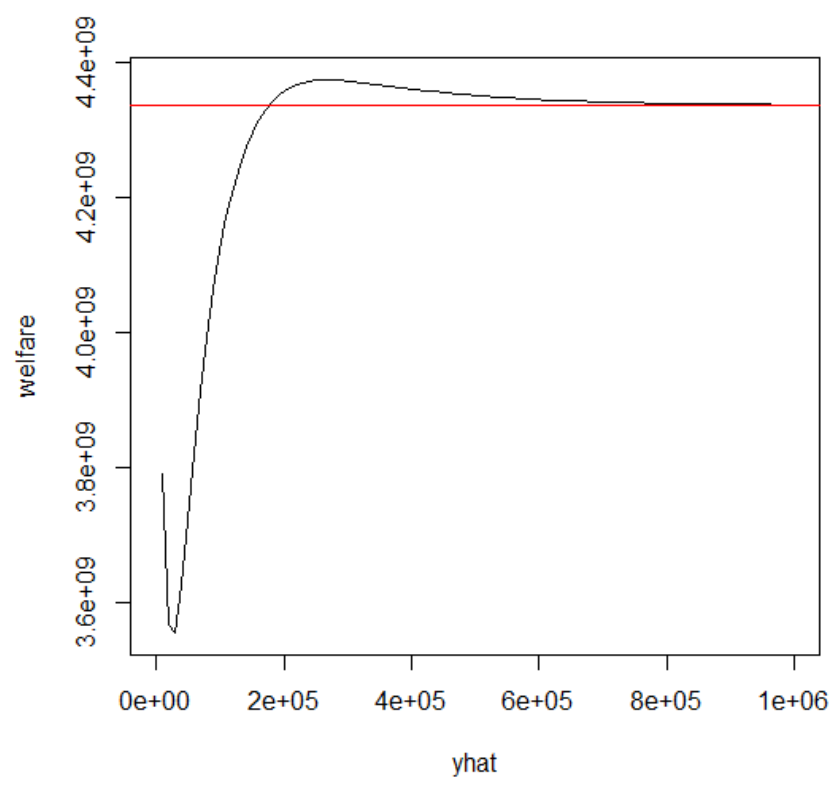

Figure 6: Welfare from sorting at cutoff $\hat{y}$ (black) and welfare without sorting (red) if $\mu=10.85$ and $\sigma=0.7$

same direction as inequality increases (in the form of a median-preserving spread) for low rates of $\sigma$. However, as can be easily demonstrated in simulations, the goals diverge for very high $\sigma$. As $\sigma$ increases above 8, the monopolist's optimal cutoff becomes much higher than the optimal cutoff for welfare, and welfare at the optimal cutoff starts to decline.

\subsection{Proof that for the atom distribution no sorting is more efficient than perfect sorting, i.e. that it has $C V \leq 1$}

Welfare from perfect sorting is given by

$$
\frac{E\left(y^{2}\right)}{2}
$$


We can calculate that

$$
E\left(y^{2}\right)=\int_{0}^{1} y^{2} f(y) d y+z=\int_{0}^{1} y^{2}(1-2 z) d y+z=\frac{1}{3}+\frac{z}{3}
$$

Therefore we see that, as described in Section $5, E\left(y^{2}\right)$ (the total surplus of perfect sorting) and welfare of perfect sorting (which is just half of it) are increasing in inequality $z$. However, welfare of perfect sorting is smaller than welfare of no sorting for all $z$ :

$$
\frac{E\left(y^{2}\right)}{2}=\frac{1}{6}+\frac{z}{6} \leq \frac{1}{4} \Longleftrightarrow z \leq 0.5
$$

Another way to see this is to calculate the coefficient of variation:

$$
C V=\frac{\sqrt{\operatorname{Var}(y)}}{E(y)}=\frac{\sqrt{\frac{1}{3}+\frac{z}{3}-\frac{1}{4}}}{\frac{1}{2}}=2 \sqrt{\frac{1}{12}+\frac{z}{3}}
$$

It is straightforward to see that $C V \leq 1 \forall z \in[0,0.5]$ and that it reaches its maximum of 1 where $z=0.5$. If $z=0.5$, perfect sorting would yield the same welfare than no sorting if the sorting fee is set at $\frac{1}{2}$, such that the total surplus is split in half. However, the sorting fee is not uniquely determined in this case and a profit-maximizing monopolist would set it as high as possible, which would be 1 in this case, such that total welfare is 0 and the monopolist gets all the surplus from sorting (which is 0.5 ) for herself.

\subsection{Proof that the house distribution is NBUE}

In order to prove that the house distribution is NBUE, I need to show that

$$
\bar{E}-E-\hat{y}<0 \quad \forall \hat{y}, \quad \forall z \in[-2,2] .
$$

If $\hat{y} \leq 0.5$ we have that

$$
\bar{E}-E-\hat{y}=\frac{6-6 \hat{y}^{2}-3 z \hat{y}^{2}+8 z \hat{y}^{3}}{12-12 \hat{y}-6 z \hat{y}+12 z \hat{y}^{2}}-\frac{1}{2}-\hat{y}
$$




$$
=\frac{-6 \hat{y}+6 \hat{y}^{2}+z\left(-4 \hat{y}^{3}+3 \hat{y}-3 \hat{y}^{2}\right)}{12-12 \hat{y}-6 z \hat{y}+12 z \hat{y}^{2}} .
$$

The denominator is always positive, so we just need to analyze the numerator: $-6 \hat{y}+6 \hat{y}^{2}$ is always negative, and $-4 \hat{y}^{3}+3 \hat{y}-3 \hat{y}^{2}$ is positive for $\hat{y} \leq 0.5$, hence if $z$ is negative, the whole expression is negative for sure. If $z$ is positive, then the numerator reaches its maximum at $z=2$, where it becomes $-6 \hat{y}+6 \hat{y}^{2}-8 \hat{y}^{3}+6 \hat{y}-6 \hat{y}^{2}=-8 \hat{y}^{3}$ which is always negative. Hence, $\bar{E}-E-\hat{y}<0$ if $\hat{y} \leq 0.5$.

If $\hat{y} \geq 0.5$ we have that

$$
\begin{gathered}
\bar{E}-E-\hat{y}=\frac{6-z-6 \hat{y}^{2}+9 z \hat{y}^{2}-8 z \hat{y}^{3}}{12-6 z-12 \hat{y}+18 z \hat{y}-12 z \hat{y}^{2}}-\frac{1}{2}-\hat{y} \\
=\frac{-6 \hat{y}+6 \hat{y}^{2}+z\left(2+4 \hat{y}^{3}-3 \hat{y}-3 \hat{y}^{2}\right)}{12-6 z-12 \hat{y}+18 z \hat{y}-12 z \hat{y}^{2}} .
\end{gathered}
$$

The denominator is again positive, and the first term of the numerator, $-6 \hat{y}+6 \hat{y}^{2}$ is always negative. $2+4 \hat{y}^{3}-3 \hat{y}-3 \hat{y}^{2}$ reaches its minimum at $\frac{1}{4}+\sqrt{\frac{5}{16}}$ where it is negative, and hence $z\left(2+4 \hat{y}^{3}-3 \hat{y}-3 \hat{y}^{2}\right)$ is positive if $z<0$, and maximal at $z=-2$. Combined with $-6 \hat{y}+6 y^{2}$ evaluated at $\frac{1}{4}+\sqrt{\frac{5}{16}}$ the total expression is negative. $2+4 \hat{y}^{3}-3 \hat{y}-3 \hat{y}^{2}$ reaches its maximum at 0.5 where it is positive and hence $z\left(2+4 \hat{y}^{3}-3 \hat{y}-3 \hat{y}^{2}\right)$ is maximal at $z=2$. Again combined with $-6 \hat{y}+6 y^{2}$ evaluated at 0.5 the whole expression is negative. Hence $\bar{E}-E-\hat{y}<0$ if $\hat{y}>0.5$, and thus the house distribution is NBUE for all $z$.

\subsection{Proof that for the house distribution no sorting is more efficient than perfect sorting, i.e. that it has $C V \leq 1$}

Total surplus from perfect sorting is

$$
\begin{gathered}
E\left(y^{2}\right)=\int_{0}^{0.5} y^{2}\left(1+\frac{z}{2}-2 z y\right) d y+\int_{0.5}^{1} y^{2}\left(1-\frac{3 z}{2}+2 z y\right) d y \\
=\frac{1}{3}+\frac{z}{48}
\end{gathered}
$$


Hence, welfare from perfect sorting is

$$
\frac{1}{6}+\frac{z}{96}
$$

and no sorting yields higher welfare than perfect sorting iff

$$
\frac{1}{6}+\frac{z}{96}<\frac{1}{4} \Longleftrightarrow 24>16+z
$$

which is satisfied for all $z \in[-2,2]$.

Equivalently, the coefficient of variation is

$$
C V=\frac{\sqrt{\frac{1}{3}+\frac{z}{48}-\frac{1}{4}}}{\frac{1}{2}}=2 \sqrt{\frac{1}{12}+\frac{z}{48}}
$$

which is strictly smaller than 1 for all $z \in[-2,2]$.

\section{References}

[1] Abdulkadiroğlu, A., Angrist, J., \& Pathak, P. (2014). The elite illusion: Achievement effects at Boston and New York exam schools. Econometrica, 82(1), 137-196.

[2] Alesina, A., \& Angeletos, G. M. (2005). Fairness and Redistribution. American Economic Review, 960-980.

[3] Algan, Y., Do, Q. A., Dalvit, N., Le Chapelain, A., \& Zenou, Y. (2015). How Social Networks Shape Our Beliefs: A Natural Experiment among Future French Politicians. (mimeo)

[4] Andreasch, M., Fessler, P., Mooslechner, P., Schürz, M. (2013) Fakten zur Vermögensverteilung in Österreich, BMASK (Ed.), Sozialbericht 2011-2012 (Vienna 2013) 247-265.

[5] Ashok, V., Kuziemko, I., \& Washington, E. (2015). Support for Redistribution in an Age of Rising Inequality:: New stylized facts and some tentative explanations. Brookings papers on economic activity, (1), 2. 
[6] Bagwell, L. S., \& Bernheim, B. D. (1996). Veblen effects in a theory of conspicuous consumption. The American Economic Review, 349-373.

[7] Bartels, L. M. (2009). Economic inequality and political representation. The unsustainable American state, 167-96.

[8] Becker, G. S. (1974). A theory of marriage. In Economics of the family: Marriage, children, and human capital (pp. 299-351). University of Chicago Press.

[9] Benabou, R., \& Ok, E. A. (2001). Social Mobility and the Demand for Redistribution: The Poum Hypothesis. The Quarterly Journal of Economics, 116(2), 447-487.

[10] Bishop, B. (2008). The big sort. Why the Clustering of America is Tearing Us Apart. NY: Houghton Mifflin.

[11] Boisjoly, J., Duncan, G. J., Kremer, M., Levy, D. M., \& Eccles, J. (2006). Empathy or antipathy? The impact of diversity. The American economic review, 96(5), 1890-1905.

[12] Bonica, A., McCarty, N., Poole, K. T., \& Rosenthal, H. (2013). Why hasn't democracy slowed rising inequality?. The Journal of Economic Perspectives, 27(3), 103-123.

[13] Borge, L., \& Rattsoe, E. J. (2004). Income Distribution and Tax Structure: Empirical Test of the Meltzer Richard Hypothesis. European Economic Review, 48, 805-826.

[14] Burns, J., Corno, L., \& La Ferrara, E. (2013). Does interaction affect racial prejudice and cooperation? Evidence from randomly assigned peers in South Africa. (mimeo)

[15] Chetty, R., Hendren, N., Kline, P., \& Saez, E. (2014). Where is the land of opportunity? The geography of intergenerational mobility in the United States (No. w19843).

[16] Corneo, G., \& Grüner, H. P. (2000). Social limits to redistribution. The American Economic Review, 90(5), 1491-1507. 
[17] Cowell, F. A. (2000). Measurement of inequality. Handbook of income distribution, 1, 87-166.

[18] Cowell, F. (2011). Measuring inequality. OUP Oxford.

[19] Cruces, G., Perez-Truglia, R., \& Tetaz, M. (2013). Biased perceptions of income distribution and preferences for redistribution: Evidence from a survey experiment. Journal of Public Economics, 98, 100-112.

[20] Dalton, H. (1920). The measurement of the inequality of incomes. The Economic Journal, 30(119), 348-361.

[21] Damiano, E., \& Li, H. (2007). Price discrimination and efficient matching. Economic Theory, 30(2), 243-263.

[22] Dustmann, C., \& Preston, I. (2001). Attitudes to ethnic minorities, ethnic context and location decisions. The Economic Journal, 111(470), 353-373.

[23] Epple, D., \& Romano, R. E. (1998). Competition between private and public schools, vouchers, and peer-group effects. American Economic Review, 33-62.

[24] Fernandez, R., \& Rogerson, R. (2003). Equity and resources: An analysis of education finance systems. Journal of Political Economy, 111(4), 858-897.

[25] Evans, G. \& Tilley, J. (2011). Private schools and public divisions: The influence of private schooling on social attitudes and political choices. in Alison Park et al (eds.), British Social Attitudes: The 28th Report. London: Sage.

[26] Forman, B., \& Koch, C. (2012). Geographic Segregation: The Role of Income Inequality. Communities and Banking.

[27] Georgiadis, A., \& Manning, A. (2012). Spend it like Beckham? Inequality and redistribution in the UK, 1983-2004. Public choice, 151(34), 537-563. 
[28] Golub, B., \& Jackson, M. O. (2012). How Homophily Affects the Speed of Learning and Best-Response Dynamics. The Quarterly Journal of Economics, 127(3), 1287-1338.

[29] Gouveia, M., \& Masia, N. A. (1998). Does the median voter model explain the size of government?: Evidence from the states. Public Choice, 97(1-2), 159-177.

[30] Hoppe, H. C., Moldovanu, B., \& Sela, A. (2009). The theory of assortative matching based on costly signals. The Review of Economic Studies, 76(1), 253-281.

[31] Karadja, M., Mollerstrom, J., \& Seim, D. (2014). Richer (and Holier) than Thou? The Effect of Relative Income Improvements on Demand for Redistribution (No. 1050). George Mason University, Interdisciplinary Center for Economic Science.

[32] Kenworthy, L., \& McCall, L. (2008). Inequality, public opinion and redistribution. Socio-Economic Review, 6(1), 35-68.

[33] Kiatpongsan, S., \& Norton, M. I. (2014). How much (more) should CEOs make? A universal desire for more equal pay. Perspectives on Psychological Science, 9(6), 587-593.

[34] Kuziemko, I., Norton, M. I., Saez, E., \& Stantcheva, S. (2015). How elastic are preferences for redistribution? Evidence from randomized survey experiments. The American Economic Review, 105(4), 14781508 .

[35] Larcinese, V. (2005). Electoral competition and redistribution with rationally informed voters. Contributions in Economic Analysis \& Policy, $4(1)$.

[36] Levy, G. and Razin, R. (2016). The Coevolution of Segregation, Polarised Beliefs and Discrimination: The Case of Private vs. State Education (mimeo)

[37] Levy, G., \& Razin, R. (2015). Preferences over equality in the presence of costly income sorting. American Economic Journal: Microeconomics, $7(2)$, 308-337. 
[38] Matakos, K. \& Xefteris, D. (2016) Divide and Rule: Redistribution in a Model with Differentiated Candidates. forthcoming in Economic Theory

[39] Meltzer, A. H., \& Richard, S. F. (1981). A rational theory of the size of government. The Journal of Political Economy, 914-927.

[40] Meltzer, A. H., \& Richard, S. F. (1983). Tests of a rational theory of the size of government. Public Choice, 41(3), 403-418.

[41] Milanovic, B. (2000). The median-voter hypothesis, income inequality, and income redistribution: an empirical test with the required data. European Journal of Political Economy, 16(3), 367-410.

[42] Norton, M. I., \& Ariely, D. (2011). Building a better America-One wealth quintile at a time. Perspectives on Psychological Science, 6(1), $9-12$.

[43] Norton, M. I., Neal, D. T., Govan, C. L., Ariely, D., \& Holland, E. (2014). The Not-So-Common-Wealth of Australia: Evidence for a Cross-Cultural Desire for a More Equal Distribution of Wealth. Analyses of Social Issues and Public Policy, 14(1), 339-351.

[44] Pesendorfer, W. (1995). Design innovation and fashion cycles. The American Economic Review, 771-792.

[45] Piketty, T. (1995). Social mobility and redistributive politics. The Quarterly journal of economics, 551-584.

[46] Piketty, T. (2014). Capital in the Twenty-first Century. Cambridge Massachusetts: Belknap of Harvard University Press

[47] Piketty, T., Saez, E., \& Stantcheva, S. (2014). Optimal taxation of top labor incomes: A tale of three elasticities. American Economic Journal: Economic Policy, 6(1), 230-271.

[48] Rayo, L. (2013). Monopolistic Signal Provision. The BE Journal of Theoretical Economics, 13(1), 27-58. 
[49] Reardon, S. F., \& Bischoff, K. (2011). Income Inequality and Income Segregation. American Journal of Sociology, 116(4), 1092-1153.

[50] Rodriguez, F. (1999). Inequality, redistribution and rent-seeking. Department of Economics, University of Maryland, 1999b.(mimeo).

[51] Rothschild, M. \& Stiglitz, J. (1970). Increasing Risk: I. A Definition. Journal of Economic Theory, 2, 225-243.

[52] Saez, E., \& Zucman, G. (2016). Wealth Inequality in the United States since 1913: Evidence from Capitalized Income Tax Data. The Quarterly Journal of Economics, 131(2), 519-578.

[53] Savage, M. (2015). Social class in the 21st century. Penguin UK.

[54] Scervini, F. (2012). Empirics of the median voter: democracy, redistribution and the role of the middle class. The Journal of Economic Inequality, 10(4), 529-550.

[55] Veblen, T. (1899). The theory of the leisure class: An economic study in the evolution of institutions. Macmillan.

[56] Watson, T. (2009). Inequality and the measurement of residential segregation by income in American neighborhoods. Review of Income and Wealth, 55(3), 820-844.

[57] Wenglinsky, H. (2007). Are private high schools better academically than public high schools?[electronic resource]. Center on Education Policy, 2007.

[58] Windsteiger, L. (2017). The Redistributive Consequences of Segregation. Working Paper of the Max Planck Institute for Tax Law and Public Finance No. 2017-12.

[59] Windsteiger, L. (2018). Sorting in the Presence of Misperceptions. Working Paper of the Max Planck Institute for Tax Law and Public Finance No. 2018-08. 